\title{
Strain localization, strong discontinuities and material fracture: Matches and mismatches
}

\author{
J. Oliver ${ }^{\mathrm{a}, *}$, A.E. Huespe ${ }^{\mathrm{b}}$, I.F. Dias ${ }^{\mathrm{a}}$ \\ ${ }^{a}$ E.T.S. d'Enginyers de Camins, Canals i Ports, Technical University of Catalonia, Campus Nord UPC, Edifici C-1, c/Jordi Girona 1-3, 08034 Barcelona, Spain \\ ${ }^{\mathrm{b}}$ INTEC-UNL-CONICET, Güemes 3450, Santa Fe, Argentina
}

\section{A R T I C L E I N F O}

\section{Article history:}

Received 27 October 2011

Received in revised form 5 April 2012

Accepted 7 June 2012

Available online 28 June 2012

\section{Keywords:}

Computational material failure

Strain localization

Strong discontinuities

Fracture mechanics

\begin{abstract}
A B S T R A C T
The work focuses on the connections of strain localization modeling of material failure and discrete fracture mechanics. It is an attempt to give an answer to the old question of whether the finite element solutions of material failure problems based on strain localization techniques, using standard continuum stress-strain constitutive models equipped with strain softening, have physical sense as solutions of de-cohesive fracture mechanics problems. Based on some well-established links of the Continuum Strong Discontinuity Approach (CSDA) to material failure and cohesive fracture mechanics, some objective indicators are proposed to assess the quality of strain localization results. These indicators are simply derived on the basis of the inelastic strain distribution provided by the strain localization solutions and the direction of propagation of the localization band. They can be computed without knowledge of the exact fracture mechanics solution of the problem, and used as error indicators in a large variety of material failure situations. The proposed indicators are assessed, by means of their application to evaluation of a number of strain localization solutions of benchmark problems. Issues as the influence of the mesh structure and alignment, type of constitutive model and considered finite element techniques are examined. On the light of the obtained results, classical flaws in strain localization solutions, as mesh bias dependence and stress-locking are reinterpreted.
\end{abstract}

(ㄷ) 2012 Elsevier B.V. All rights reserved.

\section{Motivation}

Strain localization methods are a set of tools of computational mechanics widely used in modeling material failure. They are based on the fact that the introduction of continuum (stress-strain, $\boldsymbol{\sigma}-\boldsymbol{\varepsilon}$ ) irreversible constitutive models, equipped with strain softening, in standard solid mechanics formulations induces the concentration of the strains in a narrow band, the localization band, the phenomenon being termed strain localization [1,2]. This strain localization induces and apparent jump $\llbracket \mathbf{u} \rrbracket$ of the displacement field across both sides of the localization band, which could be thought as representative of a fracture passing along some diffuse path inside the localization band (see Fig. 1).

As for the used constitutive model and its effects on the width of the localization band, two main families can be distinguished:

(1) Standard continuum constitutive models: typically stressstrain rate independent inelastic constitutive models, equipped with (softening) stress-like and strain-like internal

\footnotetext{
* Corresponding author.

E-mail addresses: xavier.oliver@upc.edu (J. Oliver), ahuespe@intec.unl.edu.ar (A.E. Huespe), idias@Inec.pt (I.F. Dias).
}

variables $[11,29,8]$, which can be inserted into the definition of generalized standard materials [21]. In the context of finite element analyzes, the localization band resulting from those methods ideally encompasses one single row of elements (see Fig. 1(a-1)).

(2) Continuum constitutive models equipped with localization limiters: they implicitly (or explicitly) incorporate an internal characteristic length, $l_{c h}$, which determines the bandwidth of the localization band. Micro-polar [31], gradient enhanced $[5,32,20]$ and non-local $[5,23]$ are typical models of this family. In order to capture that internal length, the resulting localization band has to encompass a certain number of finite elements which, due to the small character of the involved internal length, demands many times the use of auxiliary re-meshing techniques and very fine finite elements, with the associated, and some times unaffordable, computational costs (see Fig. 1(a-2)).

The first of these families of models was initially the most used for strain localization modeling purposes, because its simplicity of implementation. However, very soon two important drawbacks were reported: (a) the lack of mesh size objectivity of the results, which strongly depends on the bandwidth of the resulting 


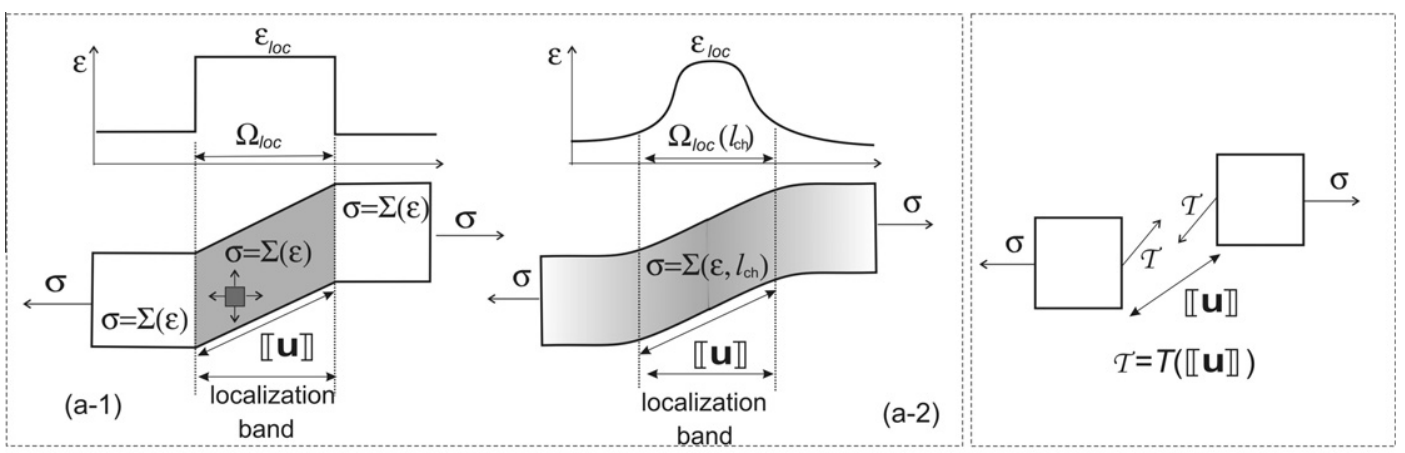

(a)

(b)

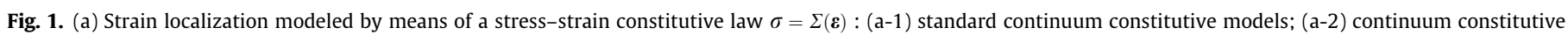
models with localization limiters and (b) discrete fracture modeled by a cohesive traction-separation law $\mathcal{T}=T(\llbracket \mathbf{u} \rrbracket)$.

localization band and, thus, of the finite element size, and (b) the lack of mesh-bias objectivity: the localization band tends to propagate following the structure of the finite element mesh and, therefore, the results strongly depend on this structure. Moreover, when the obtained localization band is not specifically aligned with the finite element mesh, the corresponding results tend to produce the so called stress locking: i.e. the stresses are not completely released as the material softens, even for high levels of the strain localization [24].

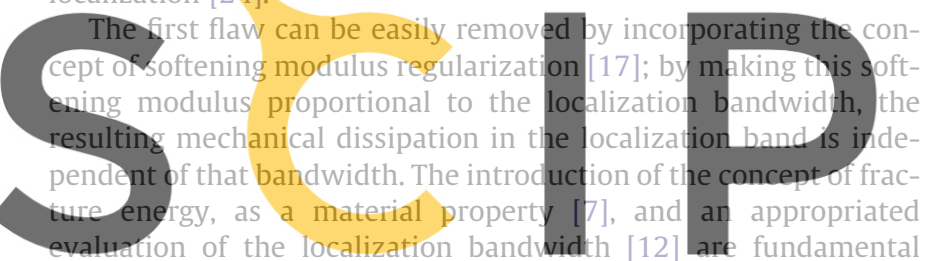
ingredients for that removal. Dealing with the second deficiency is. a much more difficult issue. The search for. remedies to this

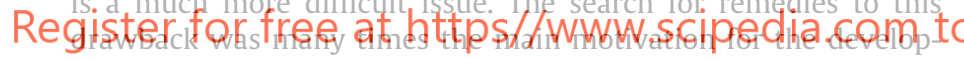

ment of the family of constitutive models equipped with localization limiters reported above, at the aforementioned cost of a large increase of the associated computational cost and, also, the introduction of, sometimes unphysical and difficult to characterize, additional parameters in the model.

Specific finite element formulations for strain localization problems have also been devised to alleviate or remove the mesh bias dependency in the first family. Mixed pressure-displacement $[4,27]$ and mixed strain-displacement [3] finite element formulations have been advocated as a way of alleviation of this flaw.

Controversy has also historically arisen about the reasons for the difficulties in strain localization modeling using standard constitutive models. For instance, it has been asserted that the reason for the observed spurious mesh bias dependence is that they lead to the "loss of ellipticity of the equilibrium rate equations... as a necessary condition for well-posedness of the rate boundary value problem" [2]. This argument has been counter-argued by stating: "this standpoint ignores the well-known fact that well-aligned finite element meshes produce good results... This evidence strongly suggests that the flaw that produces spurious mesh-bias dependence of the discrete problem is in the spatial discretization procedure" [3].

At any rate, it seems clear that the results obtained by strain localization methods are some times much better than other, that the ultimate reasons for these differences are not evident, and that procedures to grade the quality of the strain localization results are necessary.

The goal of this work is inscribed into this setting and it is twofold:
I Developing a set of indicators allowing quantification of the quality of the strain localization results, in terms of its ability to match a real fracture embedded into the localization band, and

II Proposing a specific computational mechanics framework for the development of these indicators, which can help to clarify the ultimate reasons behind the observed flaws in strain localization methods and, thus, to remove them in subsequent developments.
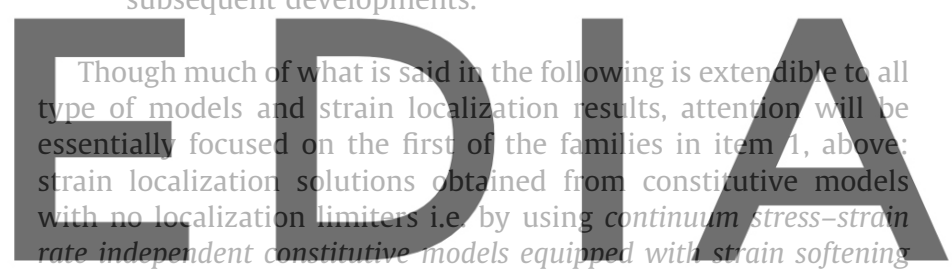

[9]. This is the case in which mesh-bias dependency is most eviclent and where the necessity to assess the quality of the results

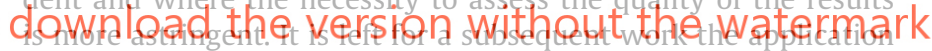
of the developed indicators to the second family of models, because, apart from the high computational cost and modeling complexity, in these models, flaws like mesh bias dependence are less evident.

The first difficulty found in achieving the goals mentioned above is to find a solid connection of strain localization with discrete fracture (see Fig. 1). In other words: to establish a link of the strain localization results, obtained in a continuum-mechanics framework using stress-strain $\boldsymbol{\sigma}-\boldsymbol{\varepsilon}$ constitutive models, with those that would be obtained if a fracture, modeled by cohesive fracture mechanics techniques (typically by traction-separation $\mathcal{T}-\llbracket \mathbf{u} \rrbracket$ models embedded into the localization band) were used. Hence, a number of relevant questions arise i.e:

- What is the traction separation law consistent with the continuum stress-strain constitutive model considered in the strain localization model?

- How the material properties of the cohesive fracture model can be related to the ones in the continuum constitutive model?, and

- What is the role, in that link, of the width of the strain localization band? (typically the finite element size)... How to make the intended identification independent of this bandwidth (that is, mesh-size objective)?

Answers to all these questions can be found in the so-called Continuum Strong Discontinuity Approach (CSDA) to material failure. This approach, developed in the 90's [28,13,15], and subsequently 
explored in the first 2000's [14,19] offers a clear link of continuum stress-strain models and cohesive fracture models. The concepts of strong discontinuity and strong discontinuity kinematics are introduced for describing a discontinuous displacement field and the corresponding strain field in a continuum mechanics setting (see Fig. 2).

The CSDA essentially shows that the insertion of a strong discontinuity kinematics into a continuum (stress-strain) constitutive model equipped with strain softening, consistently induces a tractionseparation law at the strong discontinuity path [14]. The inherited traction-separation law is a projection of the original continuum $\sigma-\boldsymbol{\varepsilon}$ model (which lives in the 3D domain $\mathcal{B}$ ) onto the 2D domain of the discontinuity interface $\mathcal{S}$, see Figs. 1 and 2(a), and inherits the irreversible character and properties of that continuum model.

Typically, in a CSDA setting, the same continuum constitutive model can be used for modeling both the bulk stress-strain relationship (during the elastic or the unloading stages) and the traction-separation law at the fracture path (in inelastic-strainsoftening stages of the material). Leaving aside the concept of strong discontinuity kinematics, this is exactly what is intended to do in strain localization models.

Therefore, the CSDA approach provides an intermediate link to establish the intended connection of strain localization with material fracture. . but it only travels the second part of the way: the connection of continuum models, in strong discontinuity settings, with cohesive fracture mechanics. The first part of the intended link, the connection of strain localization models with strong discontinuities re-

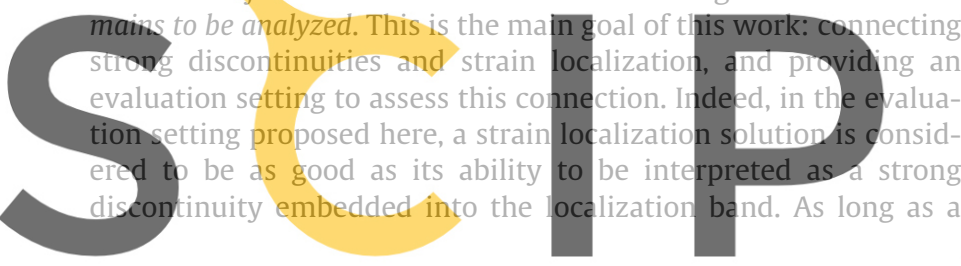

localized strain solution, obtained with a certain continuum constitutive model, matches a strong discontinuity kinematics, the CSDA supplies the remaining part of the link with fracture mechanics, and the solution can be considered representative of a fracturemechanics solution obtained with the corresponding degenerated traction-separation law.

Therefore, in this framework, the problem of the assessment of the quality of a certain localized strain solution is reduced to the qualitative and quantitative evaluation of how much the strain localization kinematics matches a strong discontinuity kinematics embedded into the localization band.

This is the basis of the proposal in this work: a diagnosis of the quality of the strain localization solution as a strong discontinuity one. If this quality is good we can consider that solution coincident with a real, physically meaningful, fracture mechanics solution but if this quality is poor the solution can be questioned. Of course, after the diagnosis, if the obtained quality is not sufficiently good, next step is to supply the remedies for the identified problems and the procedures to improve the obtained solutions. This is an issue that is not tackled here and that will be addressed in a future work. However the authors consider that an innovative approach to the identification of the ultimate reasons for those problems found in localized strain solutions, as it is done here, is not only the first step but also a very important and necessary part of its solution.

The remaining of this paper is structured as follows: in Section 2 the basics of the CSDA are recalled, the structure of a regularized strong discontinuity kinematics is compared with the strain local-

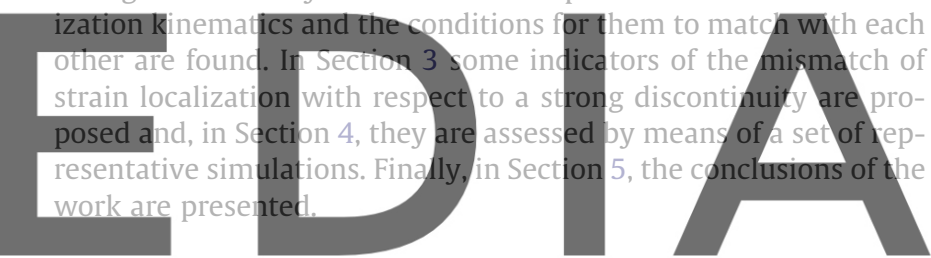

Register for free at https// AwWw_sgipedia.com to download the version ${ }^{u}$ without the watermark

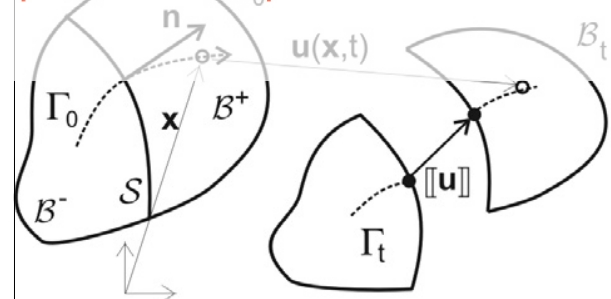

(a)

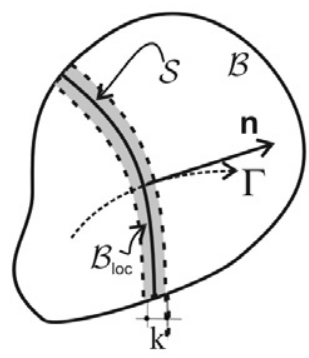

(b)
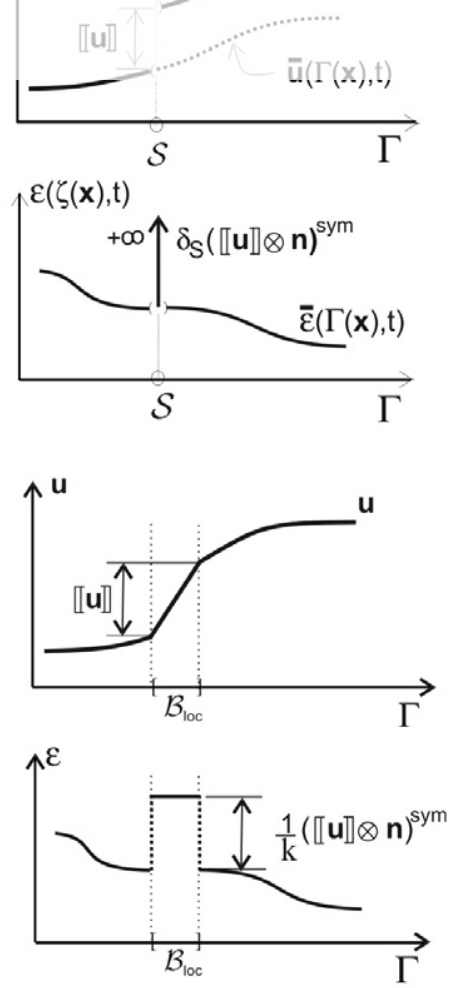

Fig. 2. (a) Strong discontinuity kinematics, (b) regularized strong-discontinuity kinematics. 


\section{The Continuum Strong Discontinuity Approach (CSDA) and strain localization}

Let us consider the body $\mathcal{B}$ in Fig. 2(a) exhibiting a strong discontinuity along the material surface $\mathcal{S}$, of unit normal $\mathbf{n}$, splitting $\mathcal{B}$ into the portions $\mathcal{B}^{+}$and $\mathcal{B}^{-}$, across which the displacement field, $\mathbf{u}(\mathbf{x}, t)$ experiences a jump of intensity $\left.\llbracket \mathbf{u} \rrbracket(\mathbf{x}, t) \equiv \mathbf{u}\right|_{\mathbf{x} \in\left(\partial \mathcal{B}^{+} \cap \mathcal{S}\right)}-$ $\left.\mathbf{u}\right|_{\mathbf{x} \in\left(\partial \mathcal{B}^{-} \cap \mathcal{S}\right)}$. The corresponding strong discontinuity kinematics defining the displacement field $\mathbf{u}(\mathbf{x}, t)$ and the strain field, $\boldsymbol{\varepsilon}(\mathbf{x}, t)$ reads [16]:

$\mathbf{u}=\overline{\mathbf{u}}+\mathcal{H}_{\mathcal{S}} \llbracket \mathbf{u} \rrbracket$
$\boldsymbol{\varepsilon} \equiv(\nabla \mathbf{u})^{\text {sym }}=\underbrace{(\nabla \overline{\mathbf{u}})^{\text {sym }}+\mathcal{H}_{\mathcal{S}}(\nabla \llbracket \mathbf{u} \rrbracket)^{s y m}}_{\overline{\boldsymbol{\varepsilon}} \text { (regular) }}+\underbrace{\delta_{\mathcal{S}}(\llbracket \mathbf{u} \rrbracket \otimes \mathbf{n})^{\text {sym }}}_{(\text {singular })}=\overline{\boldsymbol{\varepsilon}}+\delta_{\mathcal{S}}(\llbracket \mathbf{u} \rrbracket \otimes \mathbf{n})^{\text {sym }}$

where $\overline{\mathbf{u}}(\mathbf{x}, t)$ stands for the smooth part of the displacement field, $\mathcal{H}_{\mathcal{S}}(\mathbf{x})$ stands for the Heaviside function $\left(\mathcal{H}_{\mathcal{S}}(\mathbf{x})=0 \forall \mathbf{x} \in \mathcal{B}^{-}\right.$and $\left.\mathcal{H}_{\mathcal{S}}(\mathbf{x})=1 \forall \mathbf{x} \in \mathcal{B}^{+}\right)$, the notation $(\bullet)^{s y m}=\frac{1}{2}\left[(\bullet)+(\bullet)^{T}\right]$ is used for the symmetric part of $(\bullet)$ and $\delta_{S}$ stands for the Dirac's distribution shifted to $\mathcal{S}[30]$ fulfilling:

\section{$\int_{\mathcal{B}^{*}} \varphi(\mathbf{x}) \delta_{\mathcal{S}}(\mathbf{x}) d \mathcal{B}=\int_{\mathcal{S}^{*}} \varphi(\mathbf{x}) d \mathcal{S}$}

for any test function $\varphi(\mathbf{x})$ sufficiently regular and for any portion $\mathcal{B}^{*}$ of $\mathcal{B}\left(\mathcal{B}^{*} \subset \mathcal{B}\right)$ containing a portion $\mathcal{S}^{*}$ of $\mathcal{S}\left(\mathcal{S}^{*} \subset \mathcal{S}\right)$ and $\mathcal{S}^{*} \equiv\left(\mathcal{B}^{*} \cap \mathcal{S}\right)$, see Fig. 3(a). Assuming the required regularity for the term

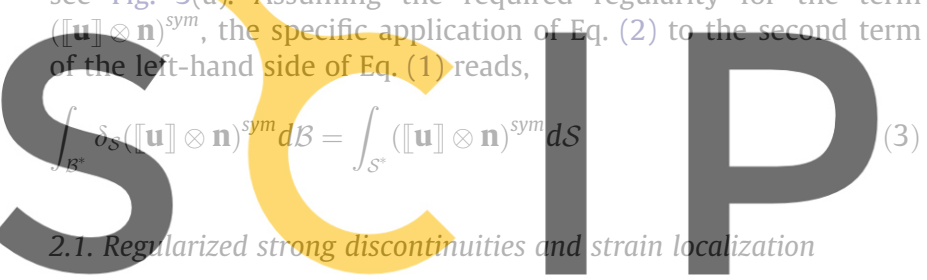

Let us now consider a finite band $\mathcal{B}_{\text {loc }}$ in $\mathcal{B}$, of very small band-

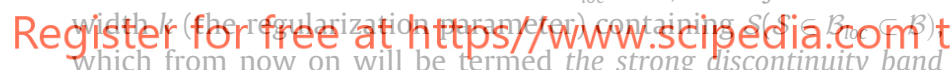
(see Figs. 2(b) and 3(b)). The Dirac's delta function in Eq. (1) can be now regularized over $\mathcal{B}_{\text {loc }}$ as $\delta_{\mathcal{S}} \rightarrow \mu_{\mathcal{B}_{\text {loc }}} \backslash k$, where $\mu_{\mathcal{B}_{\text {loc }}}$ is the colocation function on $\mathcal{B}_{\text {loc }} \quad\left(\mu_{\mathcal{B}_{\text {loc }}}(\mathbf{x})=0 \forall \mathbf{x} \notin \mathcal{B}_{\text {loc }}\right.$ and $\mu_{\mathcal{B}_{\text {loc }}}(\mathbf{x})=1 \forall \mathbf{x} \in \mathcal{B}_{\text {loc }}$ ) and $k>0$ is a (small) regularization parameter. The regularized version of Eq. (1) reads (now in rates):

$\dot{\boldsymbol{\varepsilon}} \equiv(\nabla \dot{\mathbf{u}})^{s y m}=\dot{\overline{\boldsymbol{\varepsilon}}}+\frac{\mu_{\mathcal{B}_{l o c}}}{k}(\llbracket \dot{\mathbf{u}} \rrbracket \otimes \mathbf{n})^{\text {sym }}$

so that the term $1 / k$ in Eq. (4) intensifies the strains at $\mathcal{B}_{\text {loc }}$. Thus, we can state that Eq. (4) describes a localization of the strains inside the strong discontinuity band $\mathcal{B}_{\text {loc }}$.

As $k \rightarrow 0$ then $\mathcal{B}_{\text {loc }} \rightarrow \mathcal{S}$ and $\left(\mu_{\mathcal{B}_{\text {loc }}} / k\right) \rightarrow \delta_{\mathcal{S}}$, in the sense of distributions, i.e.

$\lim _{k \rightarrow 0} \int_{\mathcal{B}^{*}} \varphi(\mathbf{x}) \frac{\mu_{\mathcal{B}_{\text {loc }}}}{k} d \mathcal{B}=\int_{\mathcal{S}^{*}} \varphi(\mathbf{x}) d \mathcal{S}=\int_{\mathcal{B}^{*}} \varphi(\mathbf{x}) \delta_{\mathcal{S}}(\mathbf{x}) d \mathcal{B}$

Therefore, we can state that the strain localization kinematics in Eq. (4) is representative (as $k \rightarrow 0$ ) of the strong discontinuity kinematics in Eq. (1). However, this is not generally true: let us consider a strain field exhibiting strain localization in a localization band $\mathcal{B}_{\text {loc }}$ (see Fig. 3(c)). In a general format its structure will be assumed to have a non-localized component, $\dot{\bar{\varepsilon}}$, and a localized component whose support is $\mathcal{B}_{l o c}$ and whose intensity is proportional to the inverse of the bandwidth $k$, i.e. ${ }^{1}$ :

\footnotetext{
${ }^{1}$ This structure is typically found in inelastic strains based models when $\dot{\overline{\boldsymbol{\varepsilon}}}=\dot{\boldsymbol{\varepsilon}}^{e}$

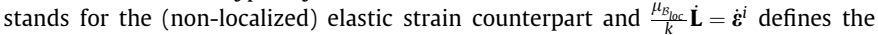
(localized) inelastic strain.
}

$$
\dot{\boldsymbol{\varepsilon}}^{l o c} \equiv(\nabla \dot{\mathbf{u}})^{\text {sym }}=\dot{\overline{\boldsymbol{\varepsilon}}}+\underbrace{\frac{\mu_{\mathcal{B}_{l o c}}}{k} \mathbf{\mathbf { L }}}_{\text {localized components }}
$$

For expressions (4) and (6) to be equivalent, $\dot{\mathbf{L}}$ is constrained by some specific conditions; in fact, comparing Eqs. (4) and (6), and taking into account Eq. (5), for they to be equivalent the following equality has to be fulfilled:

$$
\begin{aligned}
\lim _{k \rightarrow 0} \int_{\mathcal{B}^{*}} \frac{\mu_{\mathcal{B}_{\text {loc }}} \dot{\mathbf{L}} d \mathcal{B}}{k} & =\int_{\mathcal{S}^{*}} \dot{\mathbf{L}} d \mathcal{S}=\lim _{k \rightarrow 0} \int_{\mathcal{B}^{*}} \frac{\mu_{\mathcal{B}_{\text {loc }}}(\llbracket \dot{\mathbf{u}} \rrbracket \otimes \mathbf{n})^{\text {sym }} d \mathcal{B}}{k} \\
& =\int_{\mathcal{S}^{*}}(\llbracket \dot{\mathbf{u}} \rrbracket \otimes \mathbf{n})^{\text {sym }} d \mathcal{S}
\end{aligned}
$$

Taking now $\Delta \mathcal{B}^{*} \equiv \mathcal{B}^{*}$ such that the corresponding portion of discontinuity path $\Delta \mathcal{S}^{*} \equiv\left(\Delta \mathcal{B}^{*} \cap \mathcal{S}\right)$ is sufficiently small for $\mathbf{n}$ to be considered constant, Eq. (7) reads:

$$
\begin{aligned}
& \lim _{k \rightarrow 0} \int_{\Delta \mathcal{B}^{*}} \frac{\mu_{\mathcal{B}_{\text {loc }}}}{k} \mathbf{L} d \mathcal{B} \doteq \int_{\Delta S^{*}} \dot{\mathbf{L}} d \mathcal{S}=\int_{\triangle S^{*}}(\llbracket \dot{\mathbf{u}} \rrbracket \otimes \mathbf{n})^{s y m} d \mathcal{S} \\
& \left.=\left[\left(\int_{\Delta S^{*}} \llbracket \dot{\mathbf{u}}\right] d \mathcal{S}\right) \otimes \mathbf{n}\right]^{s y m} \quad \forall \Delta \mathcal{B}^{*} \subset \mathcal{B} ; \Delta \mathcal{S}^{*} \equiv\left(\Delta \mathcal{B}^{*} \cap \mathcal{S}\right)
\end{aligned}
$$

More specifically, if we consider at every point of $\mathcal{S}$ the local orthonormal basis $\left\{\hat{\mathbf{e}}_{1} \equiv \mathbf{n}, \hat{\mathbf{e}}_{2}, \hat{\mathbf{e}}_{3}\right\}$, see Fig. 3(c), the components in that basis of entities $\llbracket \dot{\mathbf{u}} \rrbracket,(\llbracket \dot{\mathbf{u}} \rrbracket \otimes \mathbf{n})^{s y m}$ and $\dot{\mathbf{L}}$ are:

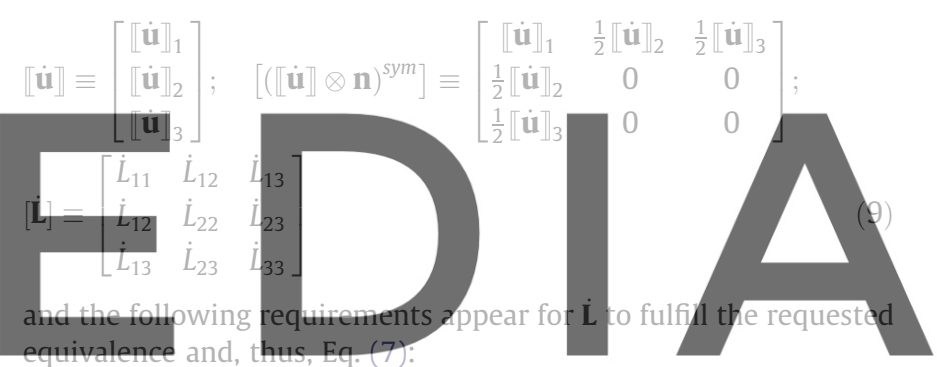

watermark

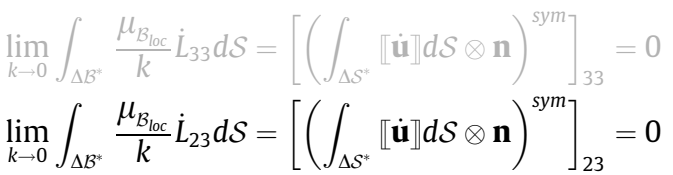

Expression (10) can be formalized to:

$\lim _{k \rightarrow 0} \int_{\Delta \mathcal{B}^{*}} \frac{\mu_{\mathcal{B}_{\text {loc }}}}{k} \dot{\mathbf{L}}_{M} d \mathcal{B}=\mathbf{0} \quad \forall \Delta \mathcal{B}^{*} \subset \mathcal{B} ; \Delta \mathcal{S}^{*} \equiv\left(\Delta \mathcal{B}^{*} \cap \mathcal{S}\right)$

with

$$
\begin{aligned}
& \dot{\mathbf{L}}_{M} \equiv \mathcal{P}_{M}: \mathbf{L}=L_{22} \hat{\mathbf{e}}_{2} \otimes \hat{\mathbf{e}}_{2}+L_{33} \hat{\mathbf{e}}_{3} \otimes \hat{\mathbf{e}}_{3}+L_{23} \hat{\mathbf{e}}_{2} \otimes \hat{\mathbf{e}}_{3}+L_{32} \hat{\mathbf{e}}_{3} \otimes \hat{\mathbf{e}}_{2} \\
& \mathcal{P}_{M} \equiv \hat{\mathbf{e}}_{2} \otimes \hat{\mathbf{e}}_{2} \otimes \hat{\mathbf{e}}_{2} \otimes \hat{\mathbf{e}}_{2}+\hat{\mathbf{e}}_{3} \otimes \hat{\mathbf{e}}_{3} \otimes \hat{\mathbf{e}}_{3} \otimes \hat{\mathbf{e}}_{3}+\hat{\mathbf{e}}_{2} \otimes \hat{\mathbf{e}}_{3} \otimes \hat{\mathbf{e}}_{2} \otimes \hat{\mathbf{e}}_{3} \\
& \quad+\hat{\mathbf{e}}_{3} \otimes \hat{\mathbf{e}}_{2} \otimes \hat{\mathbf{e}}_{3} \otimes \hat{\mathbf{e}}_{2}
\end{aligned}
$$

where $\dot{\mathbf{L}}_{M}$ will be termed the (rate of) strain localization mismatch, i.e. that part of $\dot{\mathbf{L}}$ not matching the strong discontinuity format $(\llbracket \dot{\mathbf{u}} \rrbracket \otimes \mathbf{n})^{\text {sym }}$, and $\mathcal{P}_{M}$ stands for the appropriate strain localization mismatch operator. Eq. (11) then characterizes the ability of the localized strain field (6) to represent a strong discontinuity and constitutes the basis for the intended link between strain localization and fracture.

\subsection{Strong discontinuity conditions}

Let us now consider the localized strain field in Eq. (6) and let us also assume, as it is usually done in computational material failure, that, after strain localization, the localized part of the strain, $\dot{\boldsymbol{\varepsilon}}$ corresponds to the inelastic strain, $\dot{\boldsymbol{\varepsilon}}^{(i)}$, whereas the remaining of the strain is its elastic counterpart, $\dot{\boldsymbol{\varepsilon}}^{(e)}$, being defined as: 


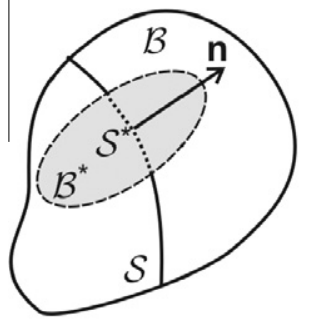

(a)

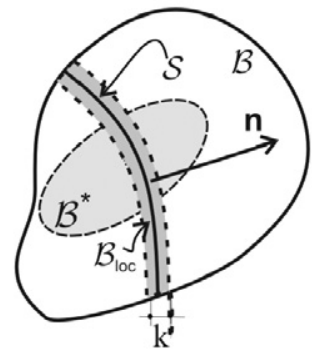

(b)

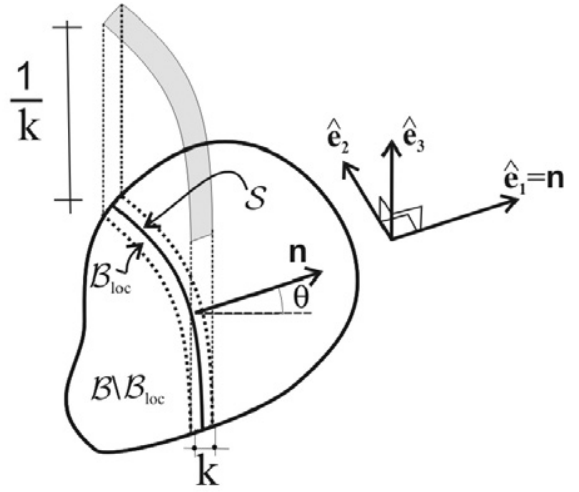

(c)

Fig. 3. (a) Strong discontinuity surface $\mathcal{S}$, (b) strong discontinuity band $\mathcal{B}_{\text {loc }}$ and (c) strain localization.

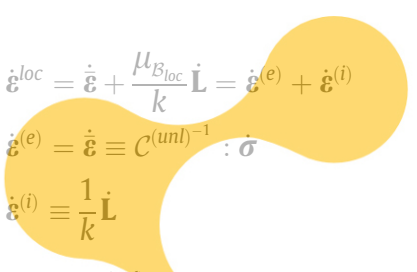

where $C^{(\text {unt) }}$ is the in-unloading tangent constitutive operator and $\sigma$
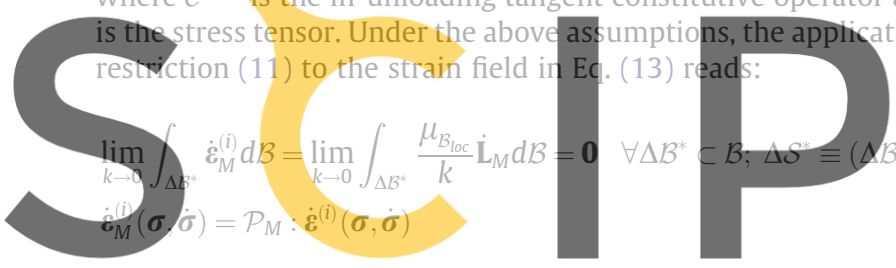

(14)

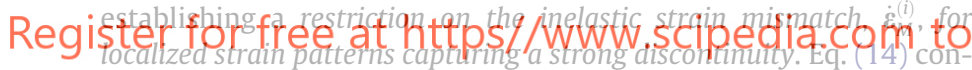

stitute the so-called strong discontinuity conditions already identified in the CSDA [15]. Eq. (14) 2 emphasizes, for standard constitutive models based on the internal variables theory, the dependence of the inelastic strain $\dot{\boldsymbol{\varepsilon}}^{i}(\boldsymbol{\sigma}, \dot{\boldsymbol{\sigma}})$ on the stress state $(\boldsymbol{\sigma})$ and on the stress evolution $(\dot{\boldsymbol{\sigma}})$. In addition, after some algebraic manipulations, Eq. (14) can be written, in a specific fashion for every family of constitutive models, only in terms of the stresses [19] as:

$\mathcal{P}_{M}: \dot{\boldsymbol{\varepsilon}}^{(i)}(\boldsymbol{\sigma}, \dot{\boldsymbol{\sigma}})=\mathbf{0} \rightarrow \mathcal{R}(\boldsymbol{\sigma})=\mathbf{0}$

this constituting a typical CSDA result indicating that strong discontinuities can only be induced for specific stress-states (fulfilling restriction (15)) at the discontinuity path. In the CSDA the so called variable bandwidth model is devised as a specific ingredient to induce such a stress state prior to the introduction of a strong discontinuity inside the localization band [16].

\section{Strain localization mismatch indicators}

Results in the previous section suggest the use of Eq. (14) to derive indicators of the quality of the strain localization patterns obtained in finite element computations.

For this purpose, let us consider the body $\mathcal{B}$ discretized in $n_{e l}$ finite elements and the set of elements $\mathcal{I}_{l o c}:=\left\{1, \ldots, n_{l o c}\right\}$, of typical size $h \equiv k$, defining the strain localization band $\mathcal{B}_{\text {loc }}^{h}$ (see Fig. 4). Let us also consider a specific element $e \in \mathcal{I}_{\text {loc }}$ of the mesh occupying the spatial domain $\mathcal{B}^{(e)} \subset \mathcal{B}_{\text {loc }}^{h}$.

The discretized version of Eq. (14) is obtained by considering $\Delta \mathcal{B}^{*} \equiv \mathcal{B}^{(e)} \subset \mathcal{B}_{\text {loc }}^{h}$ yielding:

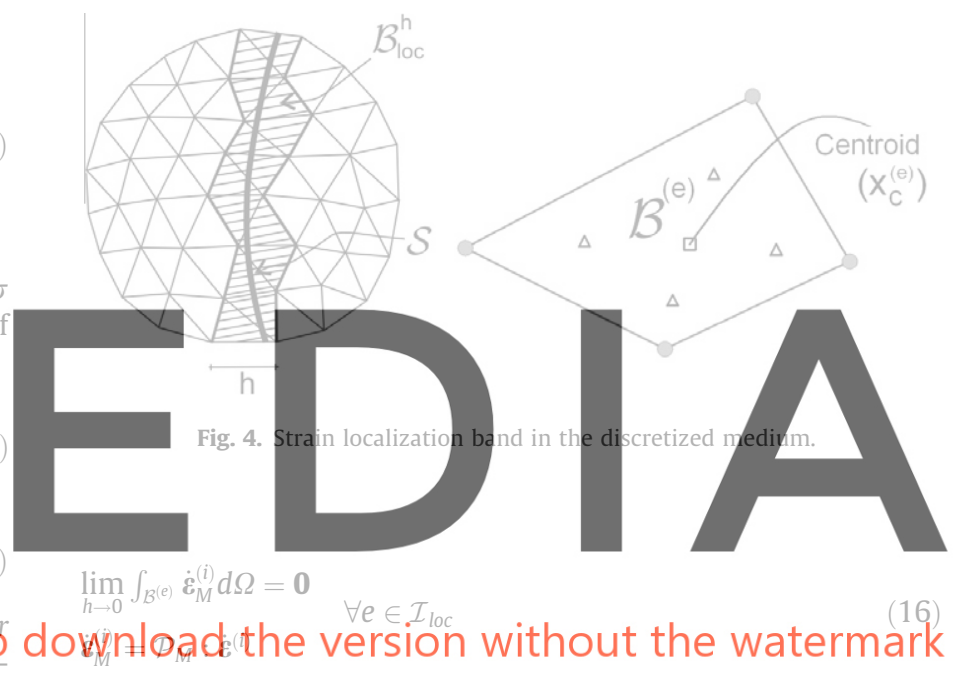

Assuming a small enough mesh size, $k \equiv h \ll \ell$ ( $\ell$ standing for a typical dimension of $\mathcal{B}$ ) Eq. (16) can be established at the asymptotic stage as:

$$
\begin{aligned}
& \int_{\mathcal{B}^{(e)}} \dot{\boldsymbol{\varepsilon}}_{M}^{(i)} d \Omega \simeq \mathbf{0} \\
& \dot{\boldsymbol{\varepsilon}}_{M}^{(i)}=\mathcal{P}_{M}: \dot{\boldsymbol{\varepsilon}}^{(i)}
\end{aligned} \quad \forall e \in \mathcal{I}_{l o c}
$$

Finally, Eq. (17) can be integrated from the bifurcation (onset of localization) time, $t_{B}^{(e)}$, to the current time, $t$, as:

$\int_{\mathcal{B}^{(e)}}\left(\int_{t_{B}^{(e)}}^{t} \dot{\boldsymbol{\varepsilon}}_{M}^{(i)} d t\right) d \Omega \simeq \mathbf{0} \quad \forall e \in \mathcal{I}_{l o c}$

\subsection{Local mismatch indicator}

Motivated by Eq. (17) the following indicator of the strain localization mismatch at element $e$ is proposed:

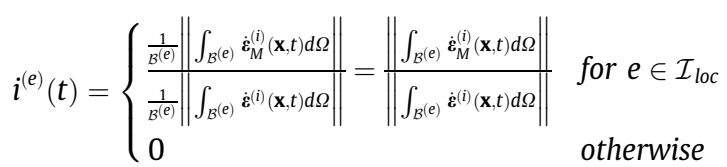

Inspection of the equation reveals that $i^{(e)}(t)$ is, at a given time $t$ and for the specific element $(e)$, the ratio of the rate of the inelastic strain mismatch and the rate of the total strain (in elemental averages and measured in Euclidean norms).

The local mismatch indicator $i^{(e)}$ displayed in Eq. (19) has the following properties: 
- For perfectly localized elements (fully representing a strong discontinuity) $i^{(e)}$ is null;

In fact, for those elements the inelastic strain $\dot{\boldsymbol{\varepsilon}}^{(i)}$ fulfills Eq. (17), which substituted in Eq. (19) returns a null value of the indicator.

- For imperfectly localized elements in $\mathcal{B}_{l o c}^{h}$ the indicator will return a value $0<i^{(e)} \leqslant 1$. The larger is the value of $i^{(e)}$ the larger is the localization mismatch of the element $e$. Therefore the map of elemental values $i^{(e)}(t)$ (in the interval $[0,1]$ ) across the finite element mesh provides a spatial description of the quality of the incremental strain localization (in terms of its match with a regularized strong discontinuity) and the distribution of the localization mismatch across the mesh. The examples presented in Section 4 illustrate this point.

- If the indicator is computed for the case of embedded strong discontinuities, E-FEM [16], it will return a null value since, again, the inelastic strain fulfills identically Eq. (17).

\subsection{Global mismatch indicators}

The local indicator in Eq. (19) provides a spatial map of the distribution, at every time of the analysis, of the localized strain mismatch over the finite element mesh. As an alternative the following global incremental strain localization mismatch indicator is proposed:

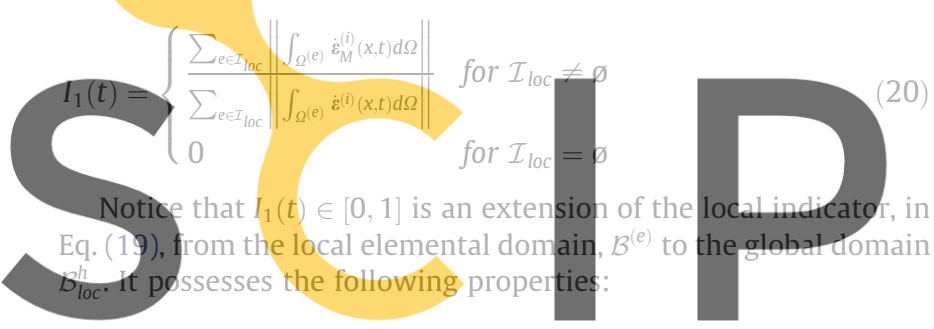

- The indicator is bandwidth objective, in the sense that, for given Register for free at htths SwW the result, at the asymptotic fact, assuming a (not necessarily perfect) localized strain field $\varepsilon^{\text {loc }}$ we can write (see Eqs. (6), (13) and (14)):

$$
\begin{aligned}
& \dot{\boldsymbol{\varepsilon}}^{l o c}=\dot{\overline{\boldsymbol{\varepsilon}}}+\frac{\mu_{\mathcal{B}_{l o c}}}{k} \dot{\mathbf{L}}=\mathcal{C}^{(\text {unl })^{-1}}: \dot{\boldsymbol{\sigma}}+\dot{\boldsymbol{\varepsilon}}^{i} \\
& \dot{\boldsymbol{\varepsilon}}^{i}=\frac{\mu_{\mathcal{B}_{l o c}}}{k} \dot{\mathbf{L}}(\boldsymbol{x}, t) ; \dot{\mathbf{L}} \cong(\llbracket \dot{\mathbf{u}} \rrbracket \otimes \mathbf{n})^{s y m} \\
& \dot{\boldsymbol{\varepsilon}}_{M}^{i}=\mathcal{P}_{M}: \dot{\boldsymbol{\varepsilon}}^{i}=\frac{\mu_{\mathcal{B}_{l o c}}}{k} \mathcal{P}_{M}: \dot{\mathbf{L}}(\boldsymbol{x}, t)=\frac{\mu_{\mathcal{B}_{l o c}}}{k} \dot{\mathbf{L}}_{M}(\boldsymbol{x}, t) ; \dot{\mathbf{L}}_{M}=\mathcal{P}_{M}: \dot{\mathbf{L}} \neq \mathbf{0}
\end{aligned}
$$

and entities in Eq. (20) can be computed, taking into account Eq. (5) as:

$$
\begin{aligned}
& \lim _{k \rightarrow 0} \int_{\mathcal{B}^{(e)}} \dot{\boldsymbol{\varepsilon}}_{M}^{(i)}(\boldsymbol{x}, t) d \Omega=\lim _{k \rightarrow 0} \int_{\mathcal{B}^{(e)}} \frac{\mu_{\mathcal{B}_{l o c}} \dot{\mathbf{L}}_{M}(\boldsymbol{x}, t) d \Omega=\int_{\mathcal{S}^{(e)}} \dot{\mathbf{L}}_{M}(\boldsymbol{x}, t) d \mathcal{S}}{k} \lim _{k \rightarrow 0} \int_{\mathcal{B}^{(e)}} \dot{\boldsymbol{\varepsilon}}^{(i)}(\boldsymbol{x}, t) d \Omega=\lim _{k \rightarrow 0} \int_{\Omega^{(e)}} \frac{\mu_{\Omega_{l o c}} \dot{\mathbf{L}}(\boldsymbol{x}, t) d \Omega=\int_{\mathcal{S}^{(e)}} \dot{\mathbf{L}}(\boldsymbol{x}, t) d \mathcal{S}}{k}
\end{aligned}
$$

where $\mathcal{S}^{(e)}=\mathcal{B}^{(e)} \cap \mathcal{S}$ stands for the portion of the discontinuity path in the element $e$. Substitution of Eq. (22) into Eq. (20) yields:

$$
\lim _{k \rightarrow 0} I_{1}(t)=\frac{\sum_{e \in \mathcal{I}_{l o c}}\left\|\int_{\mathcal{S}^{(e)}} \dot{L}_{M}(\boldsymbol{x}, t) d \mathcal{S}\right\|}{\sum_{e \in \mathcal{I}_{l o c}}\left\|\int_{\mathcal{S}^{(e)}} \dot{L}(\boldsymbol{x}, t) d \mathcal{S}\right\|}
$$

not depending on the width of the localization band $k$.

- For time steps with no localizing elements the index is null $\left(I_{1}(t)=0\right)$.

This stems from the fact that, in this case, $\mathcal{I}_{\text {loc }}=\emptyset$.

- For time steps with perfect localization in all localizing elements the index is null, since in this case $\dot{\boldsymbol{\varepsilon}}_{M}^{(i)}=\mathbf{0}$ in all elements. The same happens for the case of using embedded strong discontinuities (E-FEM approach)

- Otherwise the index returns a value $0 \leqslant I_{1}(t) \leqslant 1$.

The localization mismatch index $I_{1}(t)$ has an incremental character and informs about the quality of the localization of the rate (incre-

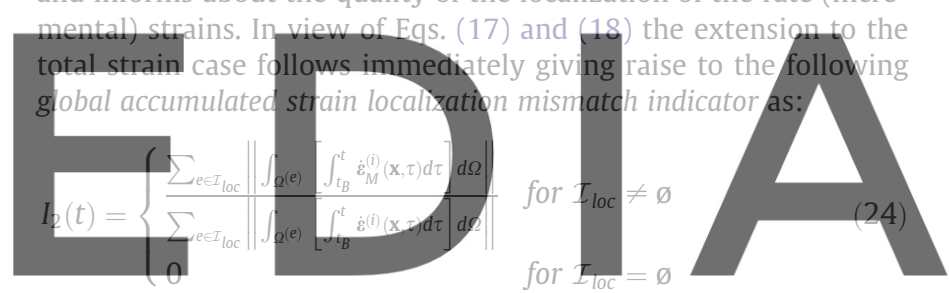

with properties similar to index $I_{1}(t)$. In Eq. (24), $t_{B}$ stands for the

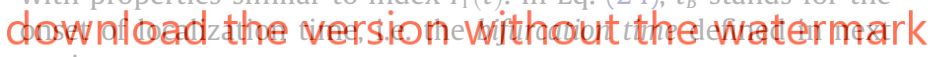
section.

3.3. Identification of the localization band and the propagation direction. Discontinuous material bifurcation analysis.

The indicators in Eqs. (19), (23) and (25) are based on the knowledge of the mismatch strain $\dot{\boldsymbol{\varepsilon}}_{M}^{(i)}$, which, in turn, requires computing the normal to the discontinuity path $\mathbf{n}$ (see Eqs. (12), (14) and Fig. 3(c), and the localization band $\mathcal{B}_{\text {loc }}$ where the
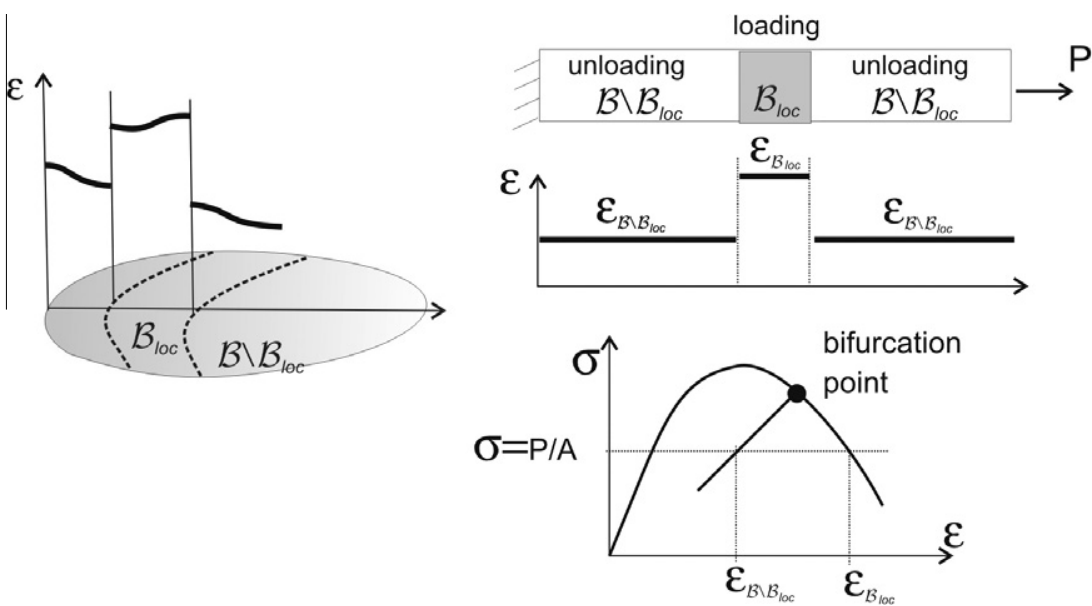

Fig. 5. Discontinuous material bifurcation problem (DMBP). 
indicators are computed. For this purpose, we resort to the discontinuous material bifurcation analysis.

The discontinuous material bifurcation problem (DMBP) is a classical one in computational material failure mechanics. In the seminal work of Hill [6] the strong ellipticity character of the, at that time termed, acoustic tensor was associated to the existence of discontinuous acceleration propagating waves. Later on, the works of Rice [22] and Rudnicki and Rice [25], among others, linked that condition to the onset of strain localization in dynamic or quasi-static problems in terms of the singularity of the, then termed, localization tensor.

In early formats, the DMBP was stated as finding the conditions for a spatially smooth stress-strain field, at a given material point $\mathbf{x}$, to bifurcate into discontinuous rate of strain fields while keeping the tractions continuous along a certain direction $\mathbf{n}$.

In the context of the CSDA the problem is stated in a slightly different way, as finding those conditions to trigger, at the bifurcation time $t_{B}(\mathbf{x})$ and at given material point $\mathbf{x}$ sustaining a smooth stressstrain field $\sigma-\varepsilon$, a regularized strong discontinuity in the format of Eq. (4) (see Fig. 5). If $\dot{\boldsymbol{\varepsilon}}_{\mathcal{B}_{\text {loc }}}\left(t_{B}\right)$ and $\dot{\boldsymbol{\varepsilon}}_{\mathcal{B} \backslash \mathcal{B}_{\text {loc }}}\left(t_{B}\right)$ stand, respectively, for the incremental rates of strain at the point and at its immediate neighborhood, according to Eq. (4) we impose the rate of strain field to have the format:

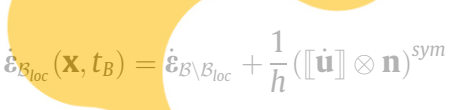

We also assume that, at the considered point, the incremental strain

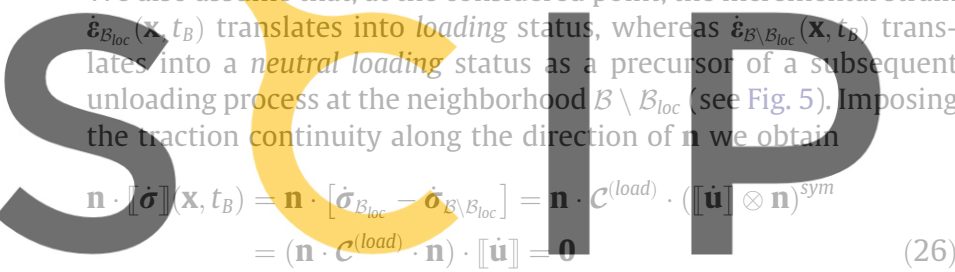

which retrieves the classical condition on the localization tensor Register for free at https//www.scipedia.com to

$\mathbf{Q}_{\text {loc }}(\boldsymbol{\sigma}, \mathbf{n}) \cdot \llbracket \dot{\mathbf{u}} \rrbracket=\left[\mathbf{n} \cdot \mathcal{C}^{(\text {load })}(\boldsymbol{\sigma}) \cdot \mathbf{n}\right] \cdot \llbracket \dot{\mathbf{u}} \rrbracket=\mathbf{0}$

as necessary for the onset of the regularized strong discontinuity.

On the basis of Eq. (27) the DMBP can be appropriately rephrased [18], in terms of the strong discontinuity mode $\mathbf{m}$, defined through $\llbracket \dot{\mathbf{u}} \rrbracket=\dot{\zeta} \mathbf{m}$, as:

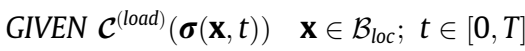

FIND the first $t \equiv t_{B} \in[0, T]$ and all $\mathbf{n} \in \mathbb{\square}:=\left\{\boldsymbol{v} \in \mathbb{R}^{3} \mid\|\boldsymbol{v}\|=1\right\}$;

$$
\begin{aligned}
\text { FULFILLING : } & \mathbf{Q}_{\text {loc, } t_{B}}(\mathbf{n}) \cdot \mathbf{m}=\left[\mathbf{n} \cdot \mathcal{C}^{(\text {load })}\left(\mathbf{x}, t_{B}\right) \cdot \mathbf{n}\right] \cdot \mathbf{m}=\mathbf{0} \\
& \text { for some } \mathbf{m} \in \mathbb{}
\end{aligned}
$$

where $[0, T]$ is the time interval of interest. Eq. (28) can be solved analytically for simple cases [26,16]. For most general cases in [18] a robust algorithm to numerically compute the solutions of Eq. (28) is provided.

In general, solving the DMBP consists of finding the bifurcation time, $t_{B}$, and a set of two solutions for the normal to the propagation direction $\left\{\mathbf{n}_{1}, \mathbf{n}_{2}\right\}$. Discriminating them is not possible just in terms of the elements of the DMBP, but, for evolving strain localization problems, this discrimination can be efficiently made as:

$$
\mathbf{n}\left(\mathbf{x}, t_{B}\right)=\underset{v \in\left\{\mathbf{n}_{1}, \mathbf{n}_{2}\right\}}{\arg \min }\left(\left|\nabla\|\dot{\mathbf{u}}\|\left(\mathbf{x}, t_{B}\right) \cdot \boldsymbol{v}\right|\right)
$$

Eq. (29) selects the normal $\mathbf{n}$ as the element of $\left\{\mathbf{n}_{1}, \mathbf{n}_{2}\right\}$ closer to the gradient of the norm of the (rate of) displacement field.

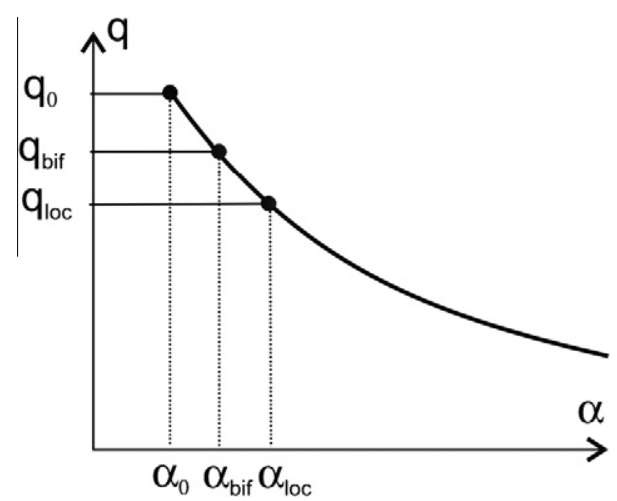

Fig. 6. Thresholds in the evolution of the stress-like/strain-like internal variables: $\left(\alpha_{0}=\alpha(\mathbf{x}, 0), q_{0}=q(\mathbf{x}, 0)\right)$ are the initial values, for $\alpha(\mathbf{x}, t), q(\mathbf{x}, t),\left(\alpha_{b i f}=\alpha\left(\mathbf{x}, t_{B}\right), q_{b i f}=q\left(\mathbf{x}, t_{B}\right)\right)$ are values at the bifurcation time, for $t=t_{B}$, and $\left(a_{l o c}=\gamma a_{b i f}\right)$ is the strain localization threshold.

Once the bifurcation time $t_{B}$ is available, the localization domain $\mathcal{B}_{\text {loc }}(t)$ is computed, at the current time $t$, as the set of material points $\mathbf{x} \in \mathcal{B}$ fulfilling the following three conditions:

(1) The material at point $\mathbf{x}$ has already bifurcated at a previous time $t_{B}(\mathbf{x}) \leqslant t$ and, therefore, it is amenable to develop a discontinuity in the strain field.

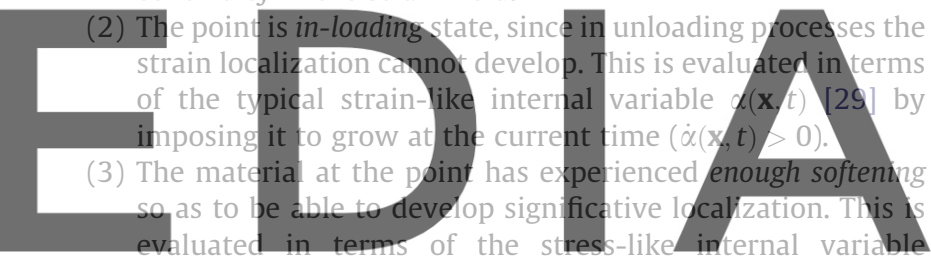

$q(\mathbf{x}, t)$ (decreasing along the deformation process for softening models, see Fig. 6), in terms of a Jocalization threshold

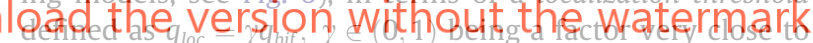
1 (typically $\gamma=0.95$ for the examples presented in Section 4 ) and $q_{\text {bif }}=q\left(\mathbf{x}, t_{B}\right)$. This keeps off the evaluation those material points with a very reduced amount of strain localization, which can produce noise in the proposed localization indicators.

For the discretized problem, $\mathbf{n}$ and $\mathcal{B}_{l o c}^{h}$ are computed by applying the previous rules to the element centroid $\mathbf{x}_{C}^{(e)}$ of every element (see Fig. 4) as

$\mathcal{B}_{\text {loc }}^{h}(t)=\cup \mathcal{B}^{(e)} \mid \dot{\alpha}\left(\mathbf{x}_{C}^{(e)}, t\right)>0, \quad q\left(\mathbf{x}_{C}^{(e)}, t\right) \leqslant \gamma q_{b i f}\left(\mathbf{x}_{C}^{(e)}\right)$

$\mathbf{n}(\mathbf{x})=\mathbf{n}\left(\mathbf{x}_{C}^{(e)}\right) \quad \forall \mathbf{x} \in \mathcal{B}^{(e)}$

\section{Representative numerical simulations}

In this section, the proposed strain localization indicators are assessed through its application to a set of well-known benchmarks. The goal is to analyze the ability of these indicators to grade the quality of the numerical solutions furnished by strain localization methods, in terms of its correlation with well-established solutions in the CSDA and cohesive fracture mechanics.

\subsection{Mode I fracture modeling using an isotropic damage model}

Fig. 7(a) shows a strip, of length $\ell=1.0 \mathrm{~m}$, undergoing horizontal stretching. The material is modeled by means of an isotropic continuum damage model [19] characterized by the elastic modulus $E=50.0[\mathrm{MPa}$, Poisson's ratio $v=0.0$, ultimate tensile stress 


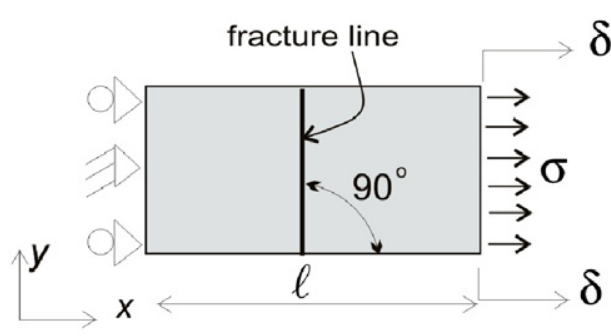

(a)

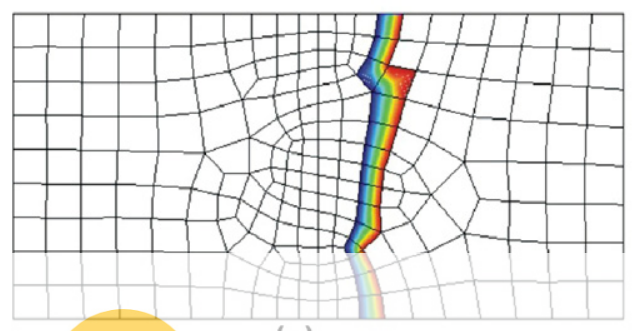

(c)

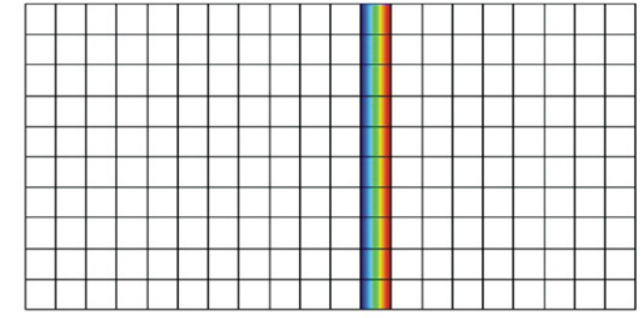

(b)

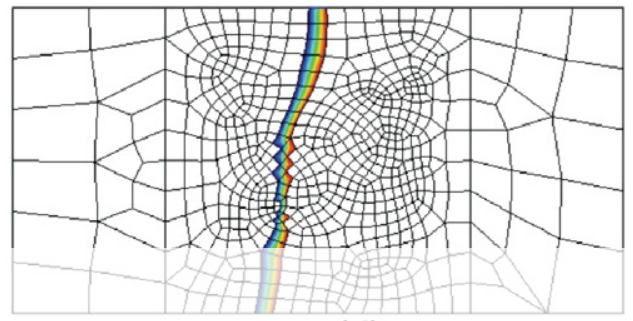

(d)

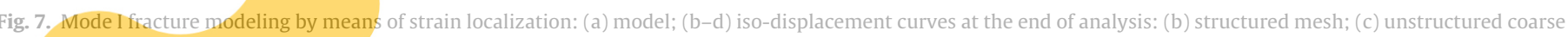
mesh; (d) unstructured fine mesh.
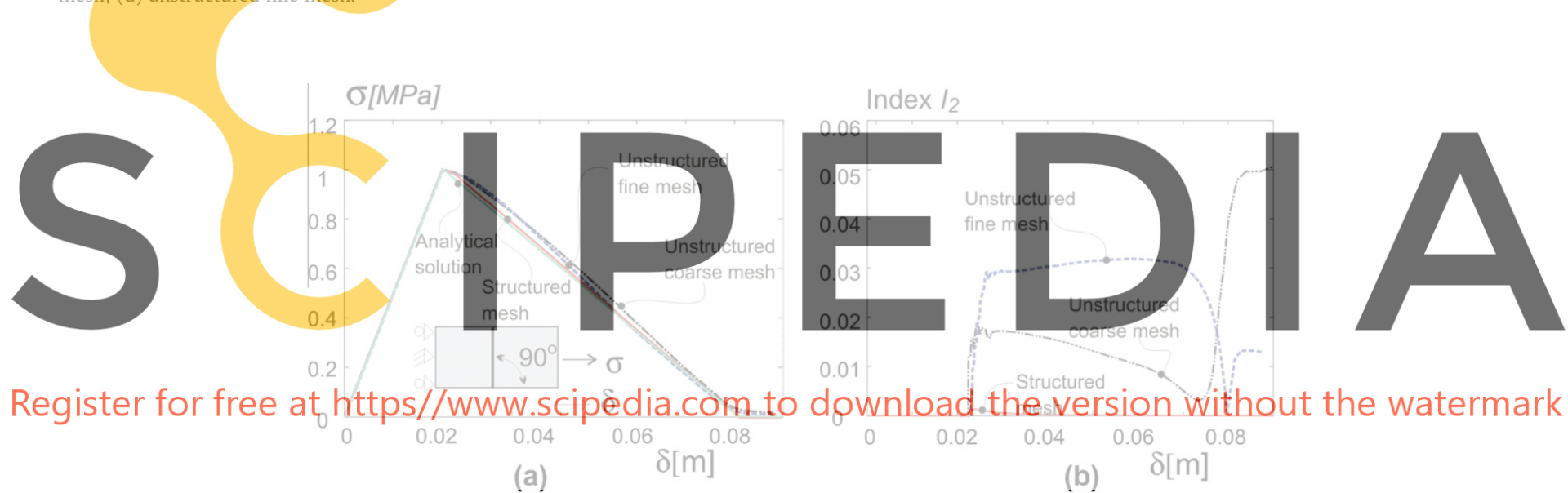

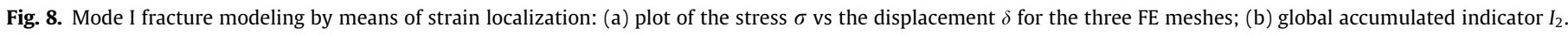

$f_{t}=1.0[\mathrm{MPa}]$ and linear softening. In order to warrant mesh size objectivity, the continuum softening modulus $H$ is regularized as $H=\bar{H} l^{(e)}$, where $\bar{H}=(1 / 2)\left(\left(f_{t}\right)^{2} /\left(E G_{f}\right)\right)$ is the intrinsic softening modulus computed in terms of the fracture energy $G_{f}=40000[\mathrm{~N} / \mathrm{m}]$ and $l^{(e)} \equiv h$ is the elemental bandwidth evaluated, for every element, as the consistent characteristic length in [12].

For these material and properties, the plane-strain problem admits an analytical solution corresponding to a vertical crack, in mode I of fracture. The structural failure of the strip is intended to be captured by means of strain localization modeling, using quadrilateral finite elements and three different finite element meshes: (1) structured mesh, uniformly-sized and vertically oriented, (see Fig. 7(b)), (2) unstructured and irregularly-sized coarse mesh (see Fig. 7(c)) and (3) unstructured and irregularly-sized fine mesh (see Fig. 7(d)). In all cases, the ultimate stress has been slightly perturbed, to $f_{t}^{*}=0.98[\mathrm{MPa}]$, in one element of the upper part of the mesh, in order to break the homogeneity of the problem and trigger the strain localization at this element. In order to check the meshbias dependence of the strain localization solution, the first mesh is thus specifically aligned with the expected crack direction, whereas the other two meshes are on purpose misaligned.
In Fig. 7(b-d) the corresponding localization bands, obtained at final stages of the simulation, are displayed by plotting the contours of the iso-displacement fields, which group inside the localization band. In all cases, the strain appears, sharply localized, in bands of one element width, but for the unstructured meshes it can be observed a clear trend to follow the mesh alignment breaking the desired vertical alignment. Indeed, in those cases a vertical crack path cannot be completely placed inside (and all along) the localization band.

The solution of the problem in terms of $\sigma-\delta$, for a monotonic increase of $\delta>0$, is independent of the strip dimensions, and it can be analytically calculated. It corresponds to the classical triangular-shaped response in Fig. 8(a), i.e. an elastic loading branch, until reaching the tensile stress $f_{t}^{*}$, followed by an inelastic softening branch, till the total stress release, and a final horizontal branch with null stress.

The obtained results for the different meshes are plotted in the same figure. The structured mesh provides an almost exact solution, only slightly deviated from the analytical one by the perturbation in the uppermost element. As for the other two unstructured meshes, the flaws of the localization direction, observed in Fig. 7, do not translate into large deviations from the exact response, as it can be checked in Fig. 8(a). However, the proposed strain 

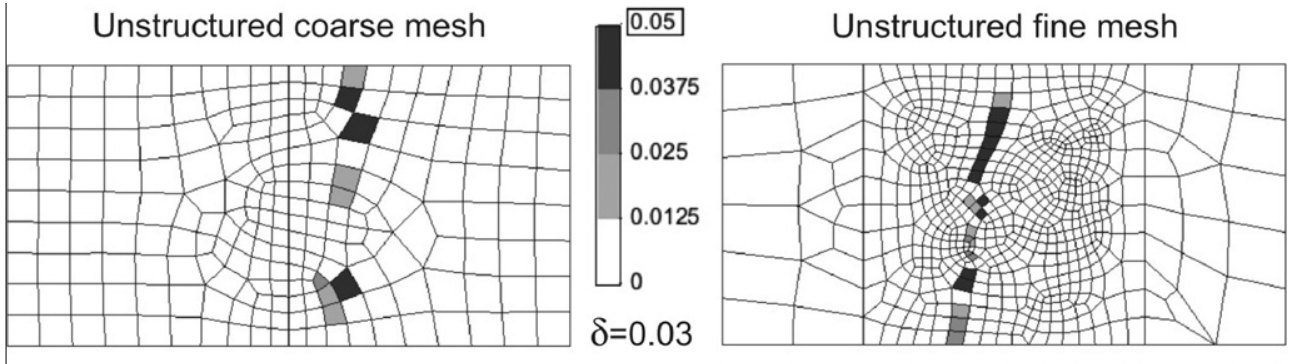

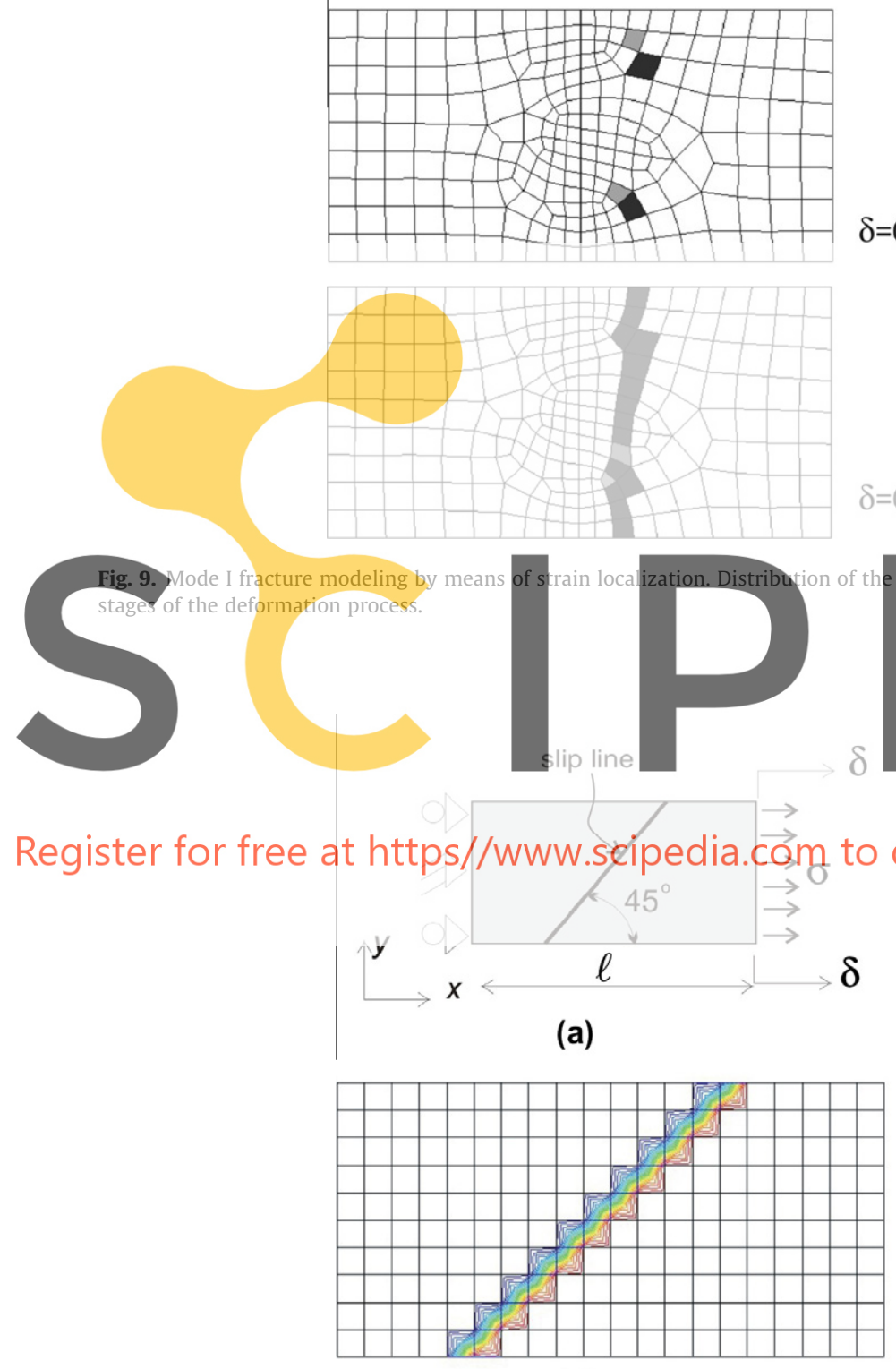

(c)
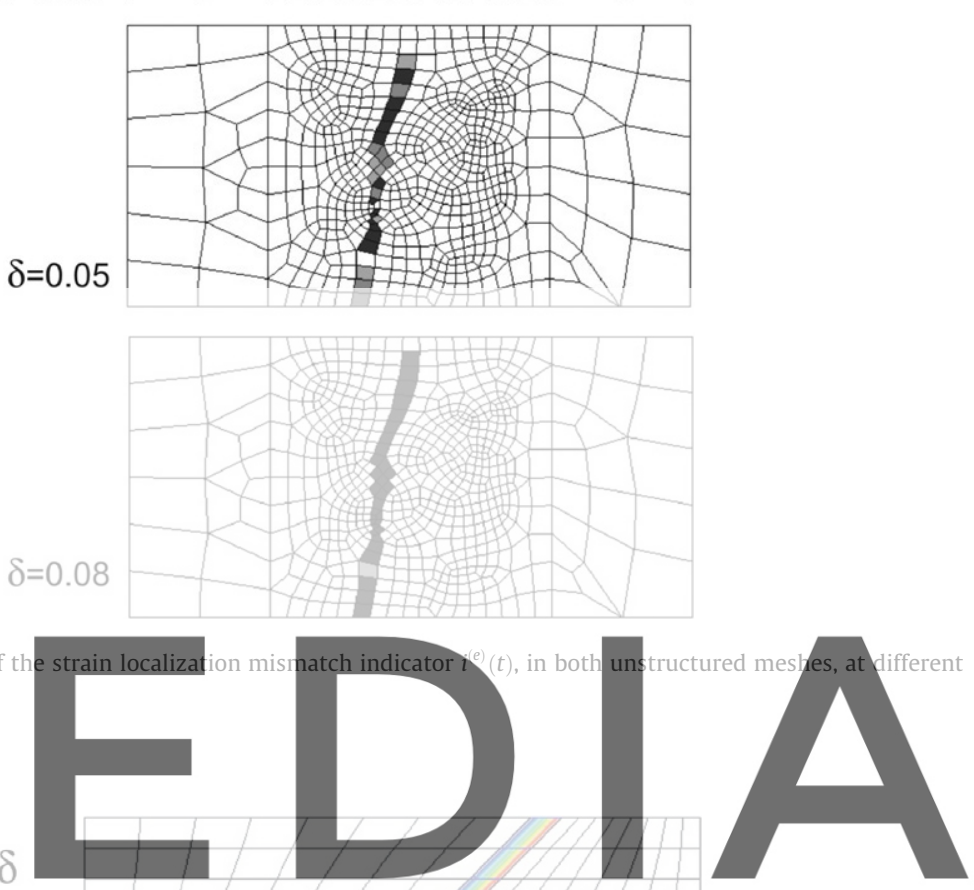

download the version without the watermark

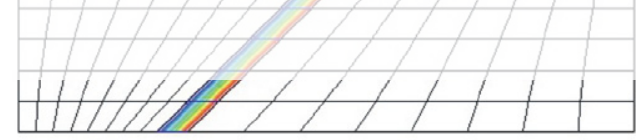

(b)

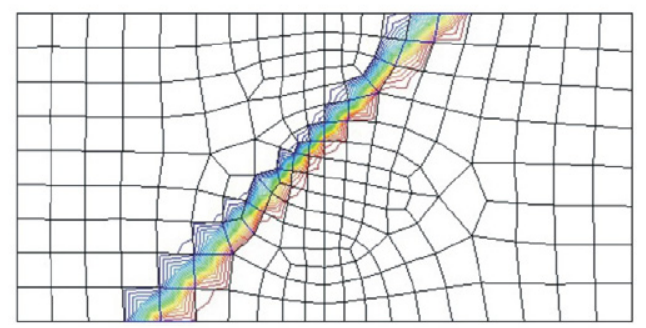

(d)

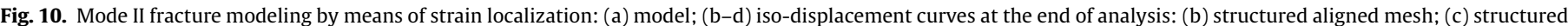
diagonal-aligned mesh; (d) unstructured mesh.

localization mismatch indicator $I_{2}$, in Eq. (24), when applied to the different meshes displays a much larger sensitivity to the deviations from the exact solution. This can be checked in Fig. 8(b): whereas for the structured vertically aligned mesh the indicator is practically null all along the simulation (only a tiny, non-zero, value is observed at the beginning of the propagation process in the vicinity of $\delta=0.02$, due to the introduced perturbation in the uppermost element of the band) the indicator is clearly non-zero for the two unstructured meshes. Moreover, the finer is the mesh the larger is the strain localization mismatch displayed by the index, this showing that refinement of unstructured meshes does not provide a convergent sequence of the solution to the right fracture representation.

Fig. 9 displays the spatial map of the distribution of the local mismatch indicator $i^{(e)}(t)$ in Eq. (19), at different stages of the analysis, characterized by the displacement $\delta$, for the two unstructured 


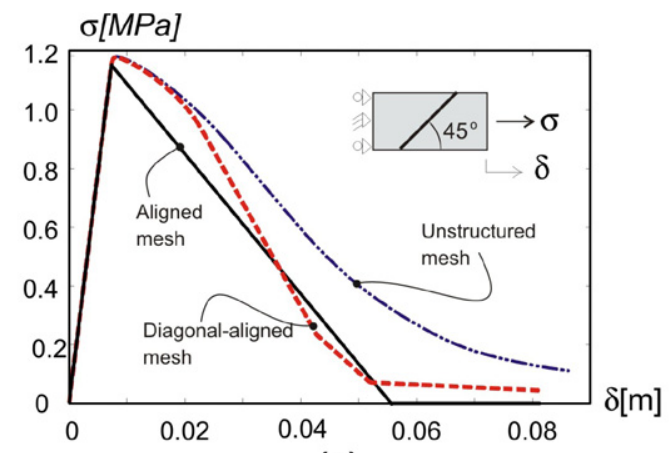

(a)
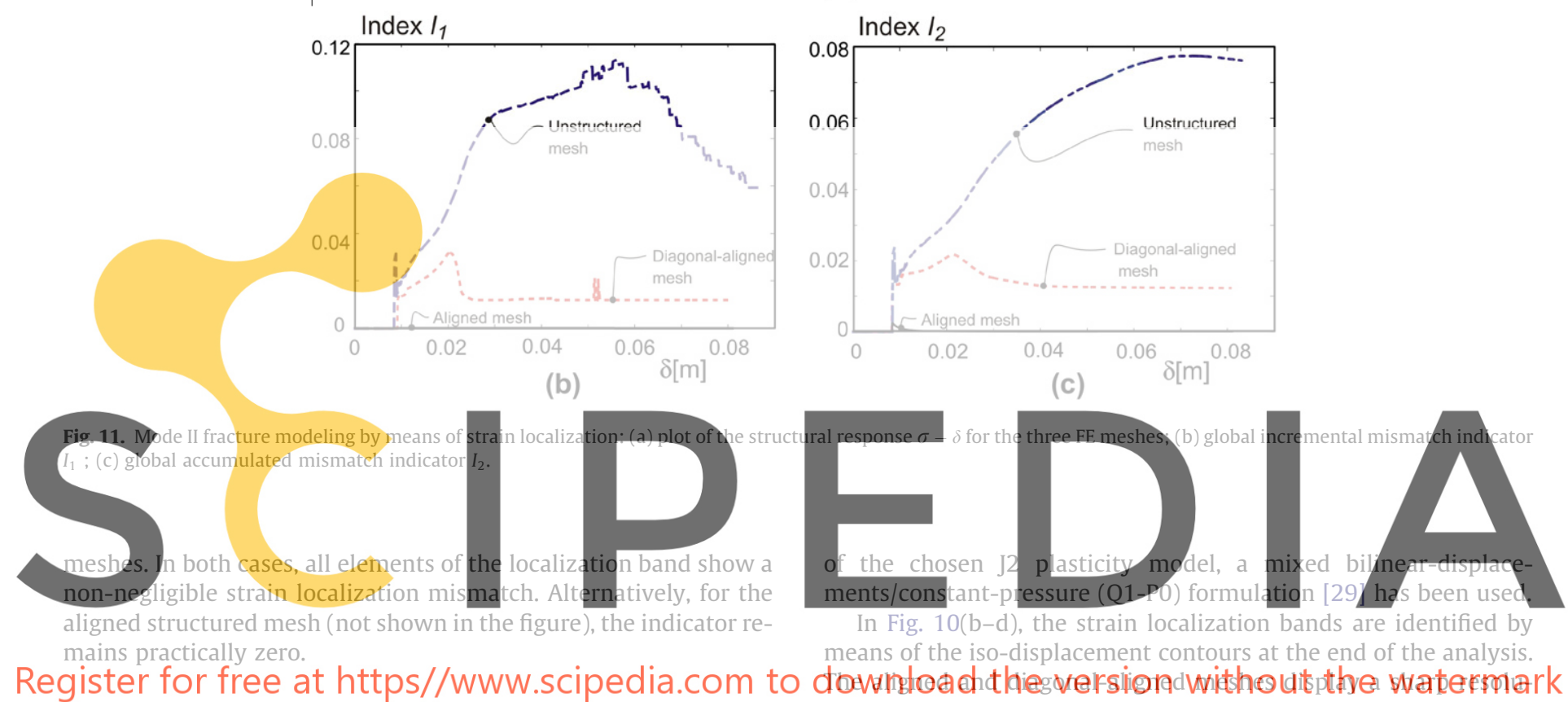

In Fig. 10(b-d), the strain localization bands are identified by means of the iso-displacement contours at the end of the analysis.

Register for free at https//www.scipedia.com to

\subsection{Mode II fracture modeling using a J2 plasticity model}

The same strip problem is now considered in Fig. 10 by modifying the constitutive behavior of the material to a J2 plasticity model. The material properties are: elastic modulus $E=120.0[\mathrm{MPa}]$, Poisson's ratio $v=0.49$, yield stress $\sigma_{y}=1.00[\mathrm{MPa}]$ and linear softening, with continuum softening modulus regularized as $H=\bar{H} l^{(e)}$, where $\bar{H}=(1 / 2)\left(\sigma_{y}^{2} / G_{f}\right)$ is the intrinsic softening modulus computed in terms of the fracture energy $G_{f}=22727.0[\mathrm{~N} / \mathrm{m}]$. For the stated quasi-incompressible plain strain problem, subjected to the corresponding biaxial stress state, the analytical solution displays a mode II fracture characterized by a straight slip-line (with only tangential component in the displacement jump) crossing the strip at $45^{\circ}$.

In order to model the problem trough a strain localization process, the three finite element meshes of quadrilateral elements, in Fig. 10, are considered: (1) a structured mesh in which the central row of elements is exactly aligned with the inclination of the slip line intended to be captured (Fig. 10)(b)), Fig. 2) a mesh of squared elements structured in such a way that the diagonals of the elements are aligned with the $45^{\circ}$ inclination of the slip line (Fig. 10(c)) and, finally, an unstructured mesh with irregular mesh size (Fig. 10(d)). Again, the two uppermost elements of the corresponding localization bands are slightly perturbed in the yield stress, to $\boldsymbol{\sigma}_{y}^{*}=0.98$, in order to break the initial homogeneity of the strip, and to induce only the $+45^{\circ}$ inclination of the two possible slip line solutions and its corresponding position in the strip. To overcome the locking effects due to the incompressible character

tion of the localization band of one element bandwidth but, for the completely unstructured mesh, the strain localization is smoother and the bandwidth encompasses more than one eiement in some places. This displays the well-known, qualitative ability of mixed quads, with J2 plasticity models, to capture slip-lines aligned with the element sides or diagonals, and the deterioration of this ability in other cases.

In Fig. 11(a) the corresponding structural responses $\boldsymbol{\sigma}-\delta$ are displayed. As expected, the solution obtained with the aligned mesh (with all elements of the localization band equally perturbed) exactly matches the expected triangular solution of the analytical response, the peak value being $\sigma=(2 / \sqrt{3}) \sigma_{y}^{*}$. The diagonal-aligned squared elements mesh separates from the theoretical solution at the beginning of the softening branch, and then approaches it at final stages of the deformation process. This initial mismatch is because, for the adopted level of perturbation, the initial bandwidth of the localization band (not shown in Fig. 10) is wider than one element, but it gradually reduces to the one element bandwidth localization band, in Fig. 10(c), at final stages. Finally, the solution for the unstructured mesh exhibits a much larger deviation from the analytical solution.

In addition, in Fig. 11, evolutions of the incremental, $I_{1}$ (Fig. 11(b)), and accumulated, $I_{2}$ (Fig. 11(c)), global strain localization mismatch indicators, in Eqs. (20) and (24), are presented. Both indicators are in good correspondence with what has been mentioned above: (1) the strain localization mismatch is null for the aligned mesh, (2) it is relatively small (but not negligible) for the diagonal-aligned mesh, and (3) is much larger for the completely unstructured mesh. This is in good accordance with the quality of the solution in terms of the 
Diagonal-aligned mesh
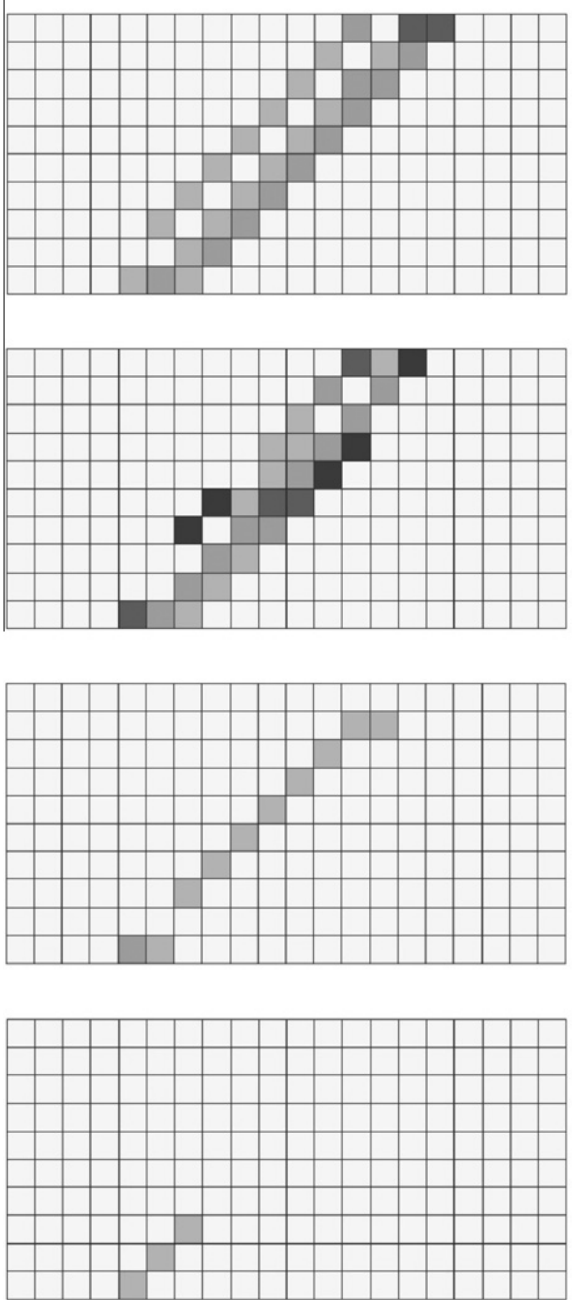

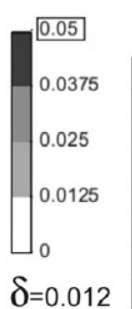

Unstructured mesh

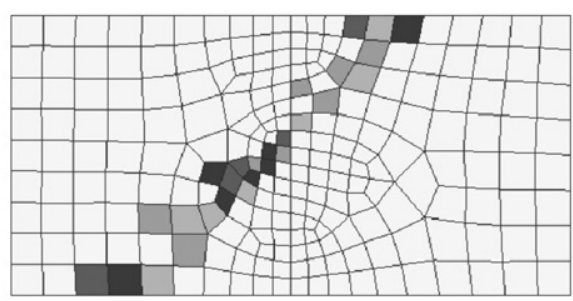

$\delta=0.022$

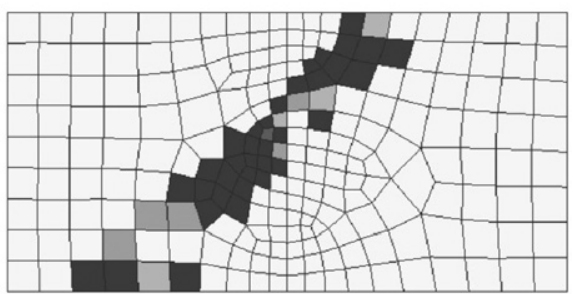

$\delta=0.040$

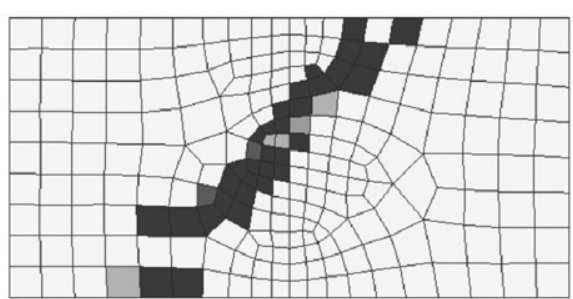

$\delta=0.080$

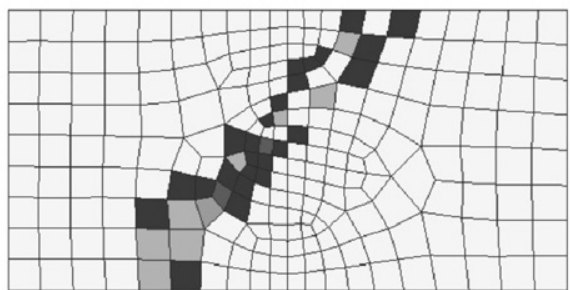

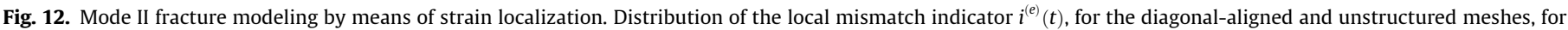
different stages of the deformation process.

sharpness of the strain localization band in Fig. 10 and of the quantitative structural responses in Fig. 11(a).

These conclusions are supplemented by the information provided in Fig. 12 in terms of the local mismatch indicator, $i^{(e)}(t)$ in Eq. (19), for different stages of the deformation process. Notice the aforementioned variation of the strain localization mismatch for the diagonal-aligned mesh, which has some relevance at stages immediately after the onset of localization displaying a band encompassing 3-4 elements. However, it almost vanishes at the ultimate stages in accordance with the sharp localization band of Fig. 10(c).

Fig. 13 displays the stress-locking level, for the diagonal-aligned and the unstructured meshes, in terms of the distribution of the elemental horizontal stress $\sigma_{x}$ that, for the theoretical fracture mechanics solution, is uniformly distributed on the strip, all along the process, and null at the end of the process. This is exactly the result obtained with the aligned mesh in Fig. 13. Notice also the correlation of the resulting distribution of the elemental stress, for the other two meshes in the figure, with the distribution of the strain localization mismatch indicator in Fig. 12.

\subsection{Propagating slip line modeling: Slope stability problem.}

In the previous examples initially near-homogenous problems, where the propagation of the localization band is almost instanta- neous, were considered. But in most cases of engineering interest, material failure takes place under heterogeneous stress states where an initial physical perturbation provokes high stress intensification in a certain point determining the onset of the failure and giving rise to a propagating localization band [10].

This is the case of next example: Fig. 14(a) describes the indentation of an embankment by a loaded footing, using a J2 plasticity model, under plain strain conditions. In a strong discontinuity solution, a slip line (mode II fracture) is triggered at a certain load level, just below the footing in the right lower corner. As the loading increases this slip-line band evolves leading to the final embankment failure by loss of its stability.

Since an analytical elasto-plastic solution is not available in this case, the numerical solution of the problem using the CSDA is considered as the reference solution. This solution consists of a slip line, which can be nearly approximated by a portion of a circle as it is shown in Fig. 14(a). Then, for the strain localization modeling purposes, a first mesh of quadrilateral finite elements is constructed approximately aligned with the theoretical slip line solution, as it is shown in Fig. 14(b), and a second misaligned mesh is considered as shown in Fig. 14(c). A J2 plasticity model is used for the material, with the following properties: elastic modulus $E=10000.0[\mathrm{MPa}$, Poisson's ratio $v=0.45$, yield stress $\sigma_{y}=100.0[\mathrm{MPa}]$ and linear softening, with continuum softening modulus regularized as 


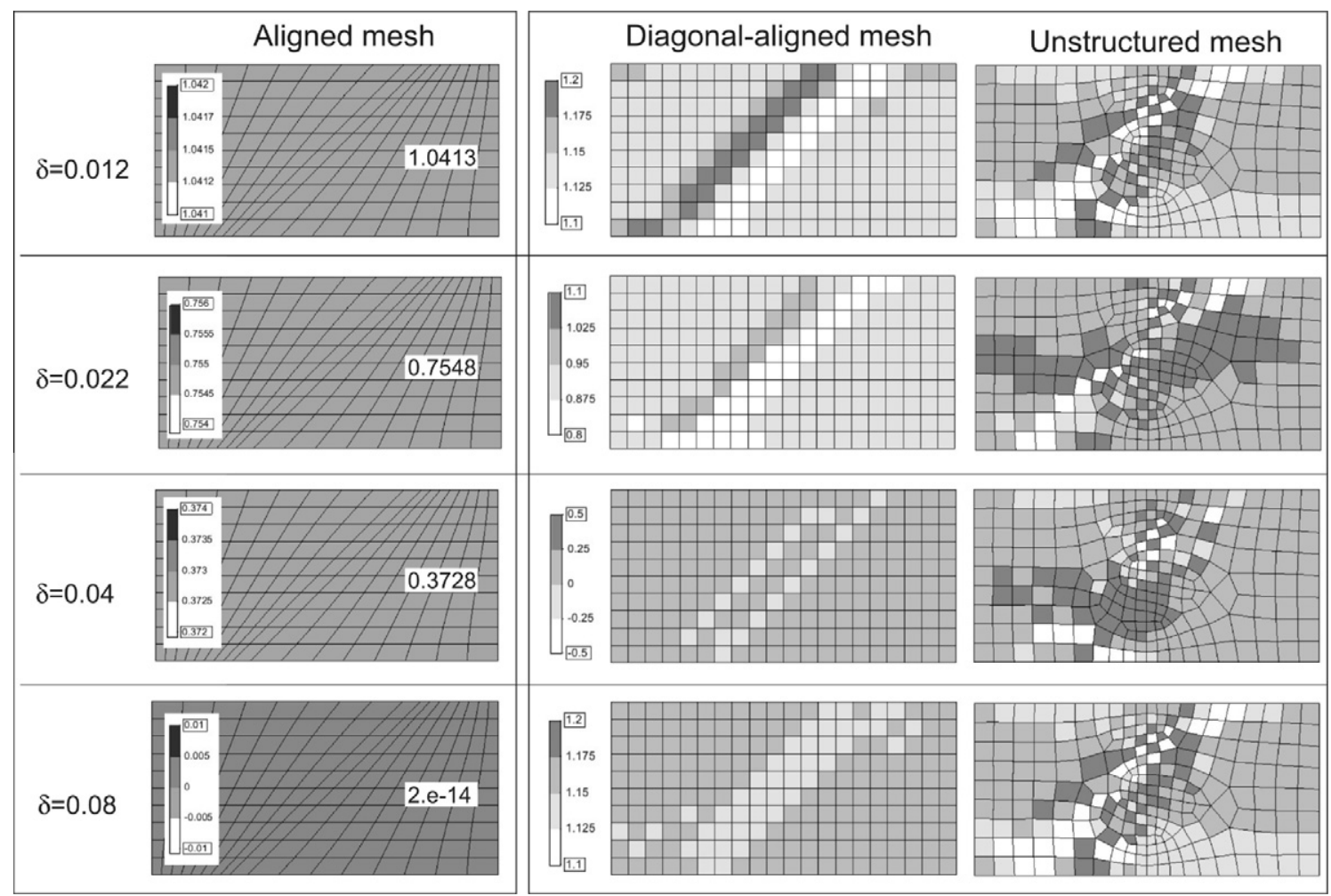

Fig. 13. Mode II fracture modeling by means of strain localization. Distribution of the horizontal stress $\sigma_{x}$, for different stages of the deformation process.

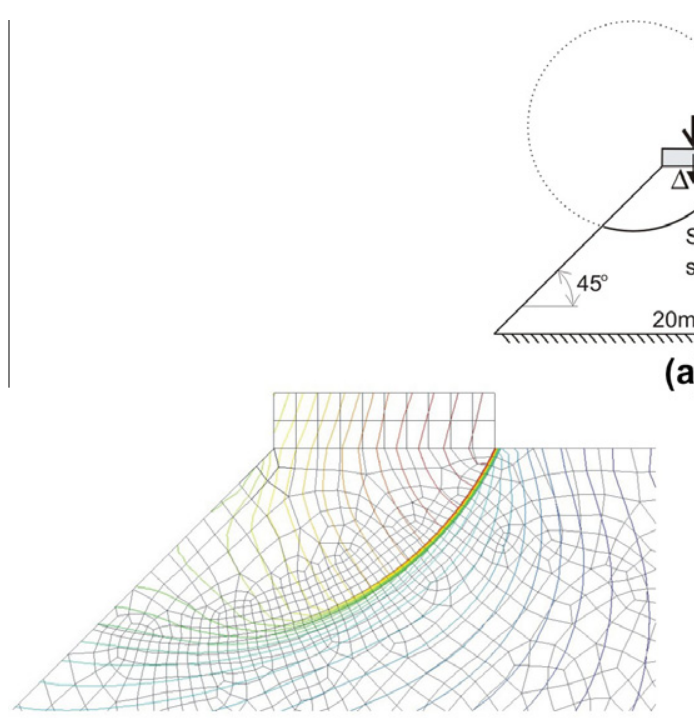

(b)

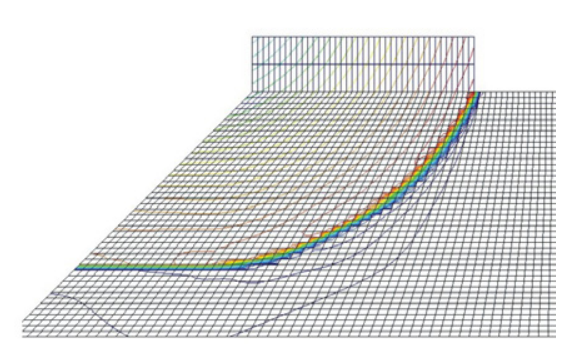

(c)

Fig. 14. Slope stability problem: (a) model; (b) aligned mesh; (c) misaligned mesh.

$H=\bar{H} l^{(e)}$, where $\bar{H}=(1 / 2)\left(\sigma_{y}^{2} / G_{f}\right)$ is the intrinsic softening modulus computed in terms of the fracture energy $G_{f}=8.0[\mathrm{MN} / \mathrm{m}]$. In order to get the solution rid of locking effects, a mixed bilinear-displacements/constant-pressure (Q1-P0) formulations has been used [29].

Fig. 14(b) and (c) display the obtained localization bands. For the aligned mesh, in Fig. 14(b), the shear band is well captured by the aligned band of elements containing the slip line: the contours of the iso-displacement field are sharply concentrated inside the portion of the, one-element-width, localization band capturing the propagating discontinuity at the considered time of the simulation. Instead, for the misaligned mesh, in Fig. 14(c), though the propagation of the discontinuity path seems to be correct, the bandwidth of the localization band is wider than one element in a large portion of it, this translating into additional dissipation of the corresponding failure mechanism.

In Fig. 15(a) the structural force-displacement, $P-\Delta$, curves are presented for the two meshes. Again, it can be observed that the misaligned mesh exhibits a substantially stiffer response as compared with the aligned mesh, which suggests an extra-dissipation, in the former, due to the strain localization mismatch. This is 


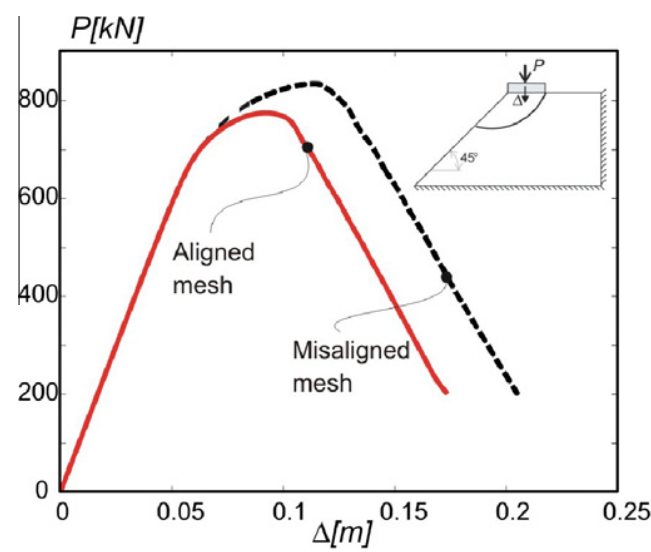

(a)

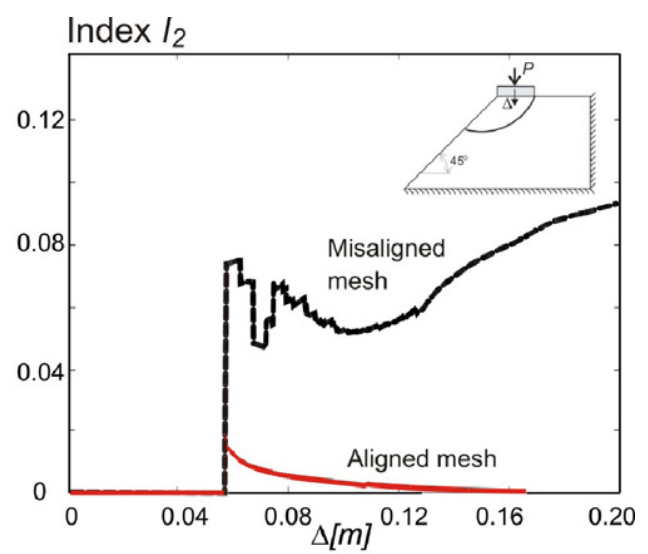

(b)

Fig. 15. Slope stability problem: (a) force-displacement structural response; (b) global accumulated strain localization mismatch indicator $I_{2}$.

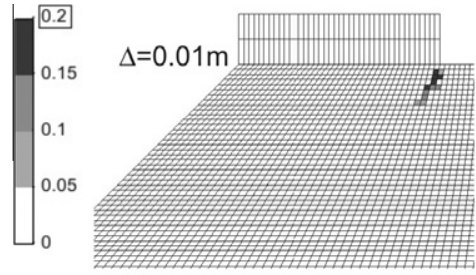

(a)

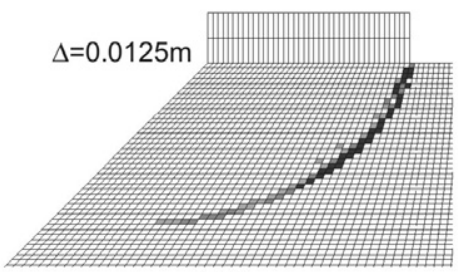

(b)

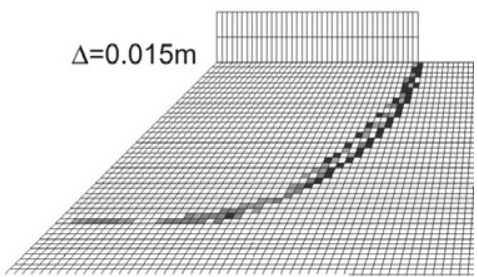

(c)

Fig. 16. Slope stability problem. Spatial distribution of the local incremental strain localization mismatch indicator mismatch $i^{(e)}(t)$, at different propagation stages.

confirmed by the indicator in Fig. 15(b) that, for the aligned mesh, is much smaller than for the unaligned mesh, but not exactly zero because a perfect alignment of the mesh with the (unknown) exact position of the slip line is not possible.

Notice that, in this case, no exact analytical solution is available; however the small values of the strain localization mismatch indicator in Fig. 15(b), suggest that, for the aligned mesh, the obtained $P-\Delta$ solution is very close to the fracture mechanics one. This shows how the proposed indicators can also be considered as error indicators with respect to fracture mechanics solutions.

Fig. 16 displays the distribution of the local incremental strain localization mismatch indicator, $i^{(e)}(t)$, for the misaligned mesh, at different stages of the strain localization propagation. The resulting spatial distribution of the indicator clearly shows as the mismatch extends to a band encompassing several elements, this indicating an imperfect representation of a discrete fracture.

\section{Concluding remarks}

Along this work, the issue of identification of strain localization solutions and the discrete fracture mechanics ones has been tackled, and some mismatch indicators to characterize the quality of the former, in terms of their ability to represent the later, have been derived. The application of such indicators to the strain localization solution of some well-established benchmarks allows arriving to the following conclusions:

- Strain localization solutions, using standard continuum constitutive models, do not always match fracture mechanics solutions. Excepting for very specific cases, typically finite element meshes specifically aligned with the (in general a priori unknown) fracture path, they exhibit a certain degree of mismatch.

- This mismatch is caused by the limited ability of standard finite elements to reproduce a regularized strong discontinuity embedded into them, this leading to the classically reported flaws of mesh-bias dependence, stress-locking etc. This inability is here identified as the ultimate reason for those flaws.

- Some constitutive models tend to produce a larger degree of mismatch than others and, in this sense, J2 plasticity models seem to be more demanding than isotropic damage models, as it can be checked from the values of the indicators in the considered examples. Also, mesh misalignment and the element size irregularity constitute sources for this mismatch, to be carefully considered when assessment of strain localization methods for modeling material fracture is addressed.

- The proposed indicators, or similar ones to be derived, can then constitute an objective tool for the quantitative evaluation of more sophisticated strain localization formulations to capture material fracture. This is an ongoing research that will be addressed in a future work.

\section{Acknowledgments}

The Spanish Ministry of Science and Innovation, and the Catalan Government Research Department, are gratefully acknowledged by the first author for their financial support to this research under Grants BIA2011-24258 and 2009 SGR 1510, respectively. The second author acknowledges the financial support from the Argentinean CONICET trough project PIP-2009 No. 0341.

\section{References}

[1] J.P. Bardet, A comprehensive review of strain localization in elastoplastic soils, Computers and Geotechnics 10 (1990) 163-188.

[2] R.d. Borst, L.J. Sluys, et al., Fundamental issues in finite element analyses of localization of deformation, Engineering Computations 10 (1993) 99-121.

[3] M. Cervera, M. Chiumenti, et al., Mixed stabilized finite element methods in nonlinear solid mechanics. Part II: strain localization, Computer Methods in Applied Mechanics and Engineering 199 (37-40) (2010) 2571-2589. 
[4] M. Cervera, M. Chiumenti, et al., Shear band localization via local J2 continuum damage mechanics, Computer Methods in Applied Mechanics and Engineering 193 (2003) 849-880.

[5] G. Pijaudier-Cabot, Z.P. Bazant, Nonlocal damage theory, Journal of Engineering Mechanics - ASCE 113 (10) (1987) 1512-1533.

[6] R. Hill, Acceleration waves in solids, Journal of the Mechanics and Physics of Solids 16 (1962) $1-10$.

[7] A. Hillerborg, Numerical methods to simulate softening and fracture of concrete, in: G.C. Sih, A.D. Tomaso (Eds.), Fracture Mechanics of Concrete: Structural Application and Numerical Calculation (1985) 141-170.

[8] A. Ibrahimbegovic, Nonlinear Solid Mechanics: Theoretical Formulations and Finite Element Solution Methods, Springer, Berlin, 2009.

[9] A. Ibrahimbegovic, D. Brancherie, Combined hardening and softening constitutive model of plasticity: precursor to shear slip line failure, Computational Mechanics 31 (1-2) (2003) 88-100.

[10] A. Kucerova, D. Brancherie, et al., Novel anisotropic continuum-discrete damage model capable of representing localized failure of massive structures. Part II: identification from tests under heterogeneous stress field, Engineering Computations 26 (1/2) (2009) 128-144.

[11] J. Lubliner, Plasticity Theory, Mcmillan Publishing Company, 1990.

[12] J. Oliver, A consistent characteristic length for smeared cracking models, International Journal for Numerical Methods in Engineering 28 (2) (1989) 461-474.

[13] J. Oliver, Modelling strong discontinuities in solid mechanics via strain softening constitutive equations. Part I. Fundamentals, International Journal for Numerical Methods in Engineering 39 (21) (1996) 3575-3600.

[14] J. Oliver, On the discrete constitutive models induced by strong discontinuity kinematics and continuum constitutive equations, International Journal of Solids and Structures 37 (48-50) (2000) 7207-7229.

[15] J. Oliver, M. Cervera, et al., Strong discontinuities and continuum plasticity models: the strong discontinuity approach, International Journal of Plasticity 15 (3) (1999) 319-351.

[16] J. Oliver, A. Huespe, Theoretical and computational issues in modelling material failure in strong discontinuity scenarios, Computer Methods in Applied Mechanics and Engineering 193 (27-29) (2004) 2987-3014.

[17] J. Oliver, A.E. Huespe, Continuum approach to material failure in strong discontinuity settings, Computer Methods in Applied Mechanics and Engineering 193 (30-32) (2004) 3195-3220.
[18] J. Oliver, A.E. Huespe, et al., On the numerical resolution of the discontinuous material bifurcation problem, International Journal for Numerical Methods in Engineering 83 (2010) 786-804.

[19] J. Oliver, A.E. Huespe, et al., From continuum mechanics to fracture mechanics: the strong discontinuity approach, Engineering Fracture Mechanics 69 (2) (2002) 113-136.

[20] R.H.J. Peerlings, R.d. Borst, et al., Gradient-enhanced damage modelling of concrete fracture, Mechanics of Cohesive Frictional Materials 3 (1998) 323-342.

[21] J. Rice, Inelastic constitutive relations for solids: an internal-variable theory and its applications to metal plasticity, Journal of the Mechancis and Physics of Solids 19 (1971) 203-240.

22] J.R. Rice, The localization of plastic deformation, in: W.T. Koiter (Ed.) Theoretical and Applied Mechanics, North-Holland Publ. Co., 1976, pp. 207220 .

[23] A. Rodrguez-Ferran, I. Morata, et al., Efficient and reliable nonlocal damage models, Computer Methods in Applied Mechanics and Engineering 193 (3032) (2004) 3431-3455.

24] J.G. Rots, Computational modeling of concrete fracture. Ph.D. Thesis, Delft University of Technology, 1988.

[25] J.W. Rudnicki, J.R. Rice, Conditions for the localization of deformation in pressure-sensitive dilatant materials, Journal of the Mechanics and Physics of Solids 23 (6) (1975) 371-394.

[26] K. Runesson, N.S. Ottosen, et al., Discontinuous bifurcations of elastic-plastic solutions at plane stress and plane strain, International Journal of Plasticity 7 (1991) 99-121.

27] PJ. Sánchez, V.E Sonzogni, et al. Study of a stabilized mixed finite element with emphasis on its numerical performance for strain localization problems, Communications in numerical methods in engineering 24 (2008) 297-320.

[28] J. Simo, J. Oliver, et al., An analysis of strong discontinuities induced by strainsoftening in rate-independent inelastic solids, Computational Mechanics 12 (1993) 277-296

[29] J.C. Simo, T.J.R. Hughes, Computational Inelasticity, Springer, 1998.

30] I. Stakgold, Green's Functions and Boundary Value Problems, Wiley, New York, 1998.

[31] P. Steinmann, K. Willam, Localization within the framework of micropola elastoplasticity, Advances in Continuum Mechanics VI (1991) 296-313.

[32] I. Vardoulakis, E.C. Aifantis, A gradient flow theory of plasticity for granular materials, Acta Mechanica 87 (1991) 197-217. 